\title{
Decays of pentaquarks in hadrocharmonium and molecular scenarios
}

\author{
Michael I. Eides ${ }^{1,2, *}$ and Victor Yu. Petrov ${ }^{2, \dagger}$ \\ ${ }^{1}$ Department of Physics and Astronomy, University of Kentucky, Lexington, Kentucky 40506, USA \\ ${ }^{2}$ Petersburg Nuclear Physics Institute, Gatchina, 188300, St.Petersburg, Russia
}

(Received 6 November 2018; published 28 December 2018)

\begin{abstract}
We consider decays of the hidden charm LHCb pentaquarks in the hadrocharmonium and molecular scenarios. In both pictures the LHCb pentaquarks are essentially nonrelativistic bound states. We develop a semirelativistic framework for calculation of the partial decay widths that allows the final particles to be relativistic. Using this approach we calculate the decay widths in the hadrocharmonium and molecular pictures. Molecular hidden charm pentaquarks are constructed as loosely bound states of charmed and anticharmed hadrons. Calculations show that molecular pentaquarks decay predominantly into states with open charm. Strong suppression of the molecular pentaquark decays into states with hidden charm is qualitatively explained by a relatively large size of the molecular pentaquark. The decay pattern of hadrocharmonium pentaquarks that are interpreted as loosely bound states of excited charmonium $\psi^{\prime}$ and nucleons is quite different. This time dominate decays into states with hidden charm, but suppression of the decays with charm exchange is weaker than in the respective molecular case. The weaker suppression is explained by a larger binding energy and respectively smaller size of the hadrocharmonium pentaquarks. These results combined with the experimental data on partial decay widths could allow to figure out which of the two theoretical scenarios for pentaquarks (if either) is chosen by nature.
\end{abstract}

DOI: 10.1103/PhysRevD.98.114037

\section{INTRODUCTION}

Pentaquarks discovered by the LHCb collaboration $[1,2]$ are the first experimental sighting of exotic baryons. It is probably not by chance that these baryons contain a heavy quark-antiquark pair, with quark masses larger than the scale of strong interactions. Internal structure of the $\mathrm{LHCb}$ pentaquarks remains at this moment unknown. Numerous models of the exotic pentaquarks were proposed in the literature, see, e.g., recent reviews [3-8] and references therein.

We will concentrate on the popular molecular and hadrocharmonium scenarios for the LHCb pentaquarks as they were realized in $[9,10]$ (see also [11]). Neither of these scenarios can be justified on purely theoretical grounds, both are based on some physically reasonable conjectures about the nature of QCD at low energies. Both in the hadrocharmonium and the molecular pictures pentaquark is assumed to be a nonrelativistic bound state of two hadrons. The main difference between the two models is in

\footnotetext{
meides@g.uky.edu

†Victor.Petrov@thd.pnpi.spb.ru
}

Published by the American Physical Society under the terms of the Creative Commons Attribution 4.0 International license. Further distribution of this work must maintain attribution to the author(s) and the published article's title, journal citation, and DOI. Funded by SCOAP . the nature of forces that bind constituents into a pentaquark. The idea of the hadrocharmonium picture [12-14] is that almost static heavy quark and antiquark inside an exotic baryon form a small color singlet state- one of excitations of charmonium. Light valence quarks inside hadrocharmonium also form a color singlet state (nucleon) and occupy a much larger volume. Interaction between an almost static color singlet heavy quark-antiquark pair and a large color singlet nucleon is due to the long range color dipole forces and effectively the small static $c \bar{c}$ pair probes the long wavelength gluon field inside the large light nucleon. Heavy quarkonium interaction with nuclei was considered in $[15,16]$, see also references in [17]. A QCD motivated potential that depends on the charmonium chromoelectric polarizability and nucleon stress-energy distribution describes charmonium-nucleon interaction, and one can find the spectrum of hidden charm baryons solving the Schrödinger equation $[9,10]$. Literally, the hadrocharmonium picture is justified in the large $N_{c}$ and heavy quark limit when the mass of the nucleon becomes large and its size remains constant, while the heavy quark-antiquark pair occupies a small volume and is effectively static $[12,13]$.

The molecular scenario of hidden charm pentaquarks initiated in [18] is qualitatively vastly different. In this scenario heavy quark and valence light quark(s) form a color singlet open charm heavy hadron, while the heavy antiquark forms another open charm hadron with the remaining light valence quark(s). These open charm 
hadrons interact via exchange of light mesons and form a loosely bound pentaquark where the open charm constituent hadrons and, respectively, heavy quark and antiquark are at rather large distances. The problem with this scenario is that meson exchanges generate attraction at large distances but are too singular at short distances and fail to hold the constituents far enough to avoid fall to the center. Some kind of hard core should arise and meson exchanges do not provide any effective repulsion at small distances. Therefore the hard core is not under theoretical control while the wave function in the molecular scenario tends to be concentrated there and critically depends on the hard core properties, see, e.g., [10] and references in the reviews $[4,5,7]$.

Currently both the molecular and hadrocharmonium descriptions of the LHCb pentaquarks are plausible, one cannot choose between them on purely theoretical grounds. Taking into account uncertainty of the theoretical situation, one needs to find experimentally observable signatures that could help to figure out which of the two scenarios (if any) is realized by nature. In principle, there are many ways to explore internal structure of hadrons, the most straightforward approach is just to measure their form factors. Information on the electromagnetic form factors of pentaquarks could immediately resolve the confrontation of the hadrocharmonium and molecular scenarios. However, one cannot expect any experimental data on the form factors of the $\mathrm{LHCb}$ pentaquarks any time soon. The next best option to explore internal structure of pentaquarks is to measure decays widths.

We expect that the dominant contributions to the total width come from two-particle decays. In the hadrocharmonium picture decays with emission of additional pions are strongly suppressed due to small phase volume and pseudoGoldstone nature of pions [9]. The constituents of the molecular pentaquark are unstable with respect to decays $D^{*} \rightarrow D+\pi$ and $\Sigma_{c} \rightarrow \Lambda_{c}+\pi$, and have finite but small widths. Three-particle decays $P_{c}(4450) \rightarrow \Sigma_{c} \bar{D} \pi$ are banned kinematically, $\quad M_{\Sigma_{c}}(2455)+M_{\bar{D}}(1865)+M_{\pi}(140)=$ $4460 \mathrm{MeV}>M_{P_{c}}(4450)$. Decays $P_{c} \rightarrow \Lambda_{c} \bar{D}^{*} \pi$ are allowed kinematically, $\quad M_{\Lambda_{c}}(2286)+M_{\bar{D}^{*}}(1865)+M_{\pi}(140)=$ $4436 \mathrm{MeV}<M_{P_{c}}$ (4450) but they are suppressed due to a small available phase volume and derivative coupling of pions.

Both in the hadrocharmonium and molecular pictures there are two qualitatively different classes of two-particle pentaquark decay processes. Decays of one kind occur without charm exchange between the constituents and the decay products carry the same charm as the constituents. In decays of the other kind charm is exchanged and the decay products have charm quantum numbers that do not coincide with the ones of the constituents.

Calculations of the pentaquark decays are impeded by numerous obstacles: apparent ultraviolet divergences, uncertainty of the cutoff momenta, need to introduce more or less arbitrary form factors, etc. We describe decay processes of nonrelativistic loosely bound pentaquarks by $t$-channel exchanges between the constituent hadrons. ${ }^{1}$ In transitions without charm exchange interaction is due to the lightest mesons without open charm. In the case when charm of the constituents changes they exchange by the lightest mesons with open charm. A naive expectation is that in each case (hadrocharmonium and molecular pentaquarks) decays without charm exchange dominate and decays with charm exchange are suppressed. This pattern of decays could allow to choose between the hadrocharmonium and molecular pictures of pentaquarks if and when the experimental data for decays will be available.

Let us quantify these expectations. Notice that to exchange charm the constituents should come very close to each other, at a relative distance $\sim 1 / m_{c}$. The probability of this to happen in a nonrelativistic bound state is proportional to $|\psi(0)|^{2} / m_{c}^{3}$, where $\psi(r)$ is the bound state wave function. But $\psi(0) \sim \kappa^{3 / 2}$, where $\kappa=\sqrt{2 \mu \epsilon}, \mu$ is the reduced mass of the system and $\epsilon$ is the binding energy. Then suppression of decays with exchange of charm is described by the factor

$$
\frac{|\psi(0)|^{2}}{m_{c}^{3}}=\left(\frac{\mu}{m_{c}}\right)^{\frac{3}{2}}\left(\frac{\epsilon}{m_{c}}\right)^{\frac{3}{2}} .
$$

In a hadrocharmonium pentaquark $\mu$ is about the nucleon mass and in a molecular pentaquark $\mu \sim m_{c}$. For the $P_{c}(4450)$ constructed in $[9,10]$ binding energy is $\epsilon \approx$ $176 \mathrm{MeV}$ in the hadrocharmonium case, and it is $\epsilon \approx$ $15 \mathrm{MeV}$ in the molecular case. At face value suppression of decays with charm exchange is expected in both pictures and it is stronger in the molecular picture. We will see below that these expectations hold and discuss what happens.

Our principal goal is to find out if measurements of partial widths for decays in the channels with open and hidden charm can help to figure our which of the two scenarios (hadrocharmonium and molecular) of the hidden charm pentaquarks is realized in nature. To this end we develop a semirelativistic approach to calculation of the decays. Let us emphasize that despite bound states both in the hadrocharmonium and the molecular pictures are nonrelativistic, loop momenta are in principle arbitrary and the final decay momentum is sometimes relativistic. In the semirelativistic approach we make a physically reasonable assumption that the intermediate virtual particles in the loop diagrams are always not far from their mass shell what allows to treat them nonrelativistically. On the other hand, our approach allows to treat the exchanged particle as well as the final particles relativistically. Below we consider decays of the hadrocharmonium and molecular pentaquarks

\footnotetext{
${ }^{1}$ Processes with the $s$-channel annihilation of heavy $c$-quarks are suppressed due to the Zweig-Okubo-Iizuka rule.
} 
from [10] in this approach. We start with the basic features of the semirelativistic approximation that allows one to calculate the pentaquark decays with a reasonable accuracy. We use Feynman diagrams to derive the interaction potentials for different decays, calculate decay widths of hadrocharmonium and molecular pentaquarks, ${ }^{2}$ make predictions for relative rates of different decays in each picture and compare the patterns of decays in hadrocharmonium and molecular scenario.

\section{SEMIRELATIVISTIC APPROXIMATION FOR PENTAQUARKS DECAYS}

\section{A. Kinematics}

The first task is to derive a practical general formula for calculation of the pentaquark decays. We consider pentaquarks as loosely bound states of two particles with binding energy $\epsilon\left(M_{P_{c}}=M_{A}+M_{B}+\epsilon\right)$ much smaller than the reduced mass of the constituents, $|\epsilon| \ll \mu=$ $M_{A} M_{B} /\left(M_{A}+M_{B}\right)$. The constituent particles are close to the mass shell and are nonrelativistic, $\epsilon / \mu \sim v^{2} / c^{2}$. In the case of the LHCb pentaquark $P_{c}(4450)$ constructed as a bound state of $\psi^{\prime}(3686)$ and the nucleon $N(940)[9,10]$ $\mu=749 \mathrm{MeV}, \epsilon=176 \mathrm{MeV}, \epsilon / \mu \sim v^{2} / c^{2} \sim 0.23$ and the relativistic correction to the binding energy is about $v^{2} /\left(4 c^{2}\right) \sim 6 \%$. The accuracy of the nonrelativistic approximation for other systems and processes considered below is roughly the same. We will use the nonrelativistic approximation in calculation of widths of loosely bound states ignoring off-mass shellness of the constituents. We expect the obtained results to have error bars about 6-8\%.

Pentaquark decays both in the hadrocharmonium and molecular pictures are due to the diagrams with the $t$-channel exchange of the type represented in Fig. 1, where $A$ and $B$ are the pentaquark constituents, and 1 and 2 are the decay products. To make the discussion more transparent we temporarily ignore spins of all particles. The final particles with masses $M_{1}$ and $M_{2}$ as well as the exchanged virtual particle $C$, could have masses significantly smaller than the masses $M_{A, B}$ of the constituents and are not necessarily nonrelativistic. We need to use relativistic kinematics for these particles. Then the decay width of the pentaquark has the form

$\Gamma=g_{1}^{2} g_{2}^{2} \frac{k}{4 \pi^{2}} \frac{E_{1} E_{2}}{M_{P_{c}}} \int d \Omega_{k}\left|\int d^{3} r e^{-i \boldsymbol{k} \cdot \boldsymbol{r}} V(\boldsymbol{r}, \boldsymbol{k}) \psi(\boldsymbol{r})\right|^{2}$,

where $\boldsymbol{k}$ is the three-momentum of the final particle 1 and we integrate over its directions, $\psi(\boldsymbol{r})$ is the normalized nonrelativistic wave function of the initial pentaquark

\footnotetext{
${ }^{2}$ Decays of pentaquarks in the molecular picture were discussed in the literature earlier, see, e.g., [19-23] and references therein. To the best of our knowledge decays in the hadrocharmonium picture were never discussed before.
}

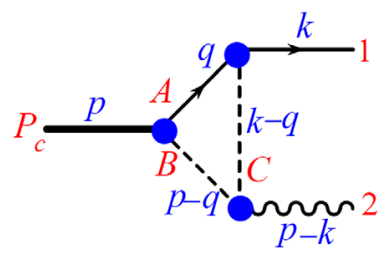

FIG. 1. Generic diagram for pentaquark decay.

(a loosely bound state of particles $A$ and $B$ ) in its rest frame, and the effective potential $g_{1} g_{2} V(\boldsymbol{r}, \boldsymbol{k})\left(g_{1,2}\right.$ are the respective coupling constants) is in the general case a function of the relative coordinate $\boldsymbol{r}$ and the final momentum $\boldsymbol{k}$. Notice the relativistic energies $E_{1,2}$ in Eq. (2) instead of the masses $M_{1,2}$ in the standard nonrelativistic formula. They arise because the final particles could be relatively light and relativistic.

The integral in Eq. (2) can be simplified when the bound state wave function $\psi(\boldsymbol{r})$ is a superposition of terms with different angular momenta $\psi(\boldsymbol{r})=\sum_{l} R_{l}(r) Y_{l m}(\theta, \phi)$ and $V(r)$ is a central potential. In such case we expand the exponential in spherical harmonics, use their orthogonality and obtain the decay amplitude as a sum of partial waves

$$
\begin{aligned}
\mathcal{M}_{i f} & =\int d^{3} r e^{-i \boldsymbol{k} \cdot \boldsymbol{r}} V(\boldsymbol{r}, \boldsymbol{k}) \psi(\boldsymbol{r}) \\
& =4 \pi \sum_{l}(-i)^{l} M(l \mid l) Y_{l m}\left(\frac{\boldsymbol{k}}{k}\right),
\end{aligned}
$$

where

$$
M(l \mid l)=\int_{0}^{\infty} r^{2} d r R_{l}(r) j_{l}(k r) V(r),
$$

and $j_{l}(k r)$ is a spherical Bessel function.

The total decay width obtained after integration over angles in this case is

$$
\Gamma=g_{1}^{2} g_{2}^{2} \frac{4 k E_{1} E_{2}}{M_{P_{c}}} \sum_{l}|M(l \mid l)|^{2} .
$$

In the calculations below the interaction potential is often a tensor, so the matrix elements similar to $M(l \mid)$ are nondiagonal in $l$, in other words orbital momentum changes in decays. The total angular momentum with account for spins is of course conserved.

The effective potential $V(\boldsymbol{r}, \boldsymbol{k})$

$$
V(\boldsymbol{r}, \boldsymbol{k})=\int \frac{d^{3} q}{(2 \pi)^{3}} e^{i \boldsymbol{q} \cdot \boldsymbol{r}} V(\boldsymbol{q}, \boldsymbol{k})
$$

can be calculated in terms of the relativistic scattering amplitude $\mathcal{A}_{A+B \rightarrow 1+2}(\boldsymbol{q}, \boldsymbol{k})$ with the nonrelativistic initial particles 


$$
g_{1} g_{2} V(\boldsymbol{q}, \boldsymbol{k})=-\frac{\mathcal{A}_{A+B \rightarrow 1+2}(\boldsymbol{q}, \boldsymbol{k})}{\sqrt{2 M_{A}} \sqrt{2 M_{B}} \sqrt{2 E_{1}} \sqrt{2 E_{2}}} .
$$

The square roots in this relationship convert the relativistically normalized scattering amplitude to the normalization used in nonrelativistic quantum mechanics. It is convenient to rescale the potential so that it coincides with the amplitude $\mathcal{A}_{A+B \rightarrow 1+2}(\boldsymbol{q}, \boldsymbol{k})$

$$
V(\boldsymbol{q}, \boldsymbol{k}) \rightarrow \frac{V(\boldsymbol{q}, \boldsymbol{k})}{\sqrt{2 M_{A}} \sqrt{2 M_{B}} \sqrt{2 E_{1}} \sqrt{2 E_{2}}} .
$$

Then the total width in Eq. (5) acquires the form

$$
\Gamma=g_{1}^{2} g_{2}^{2} \frac{4 k E_{1} E_{2}}{M_{P_{c}}} \frac{\sum_{l}|M(l \mid l)|^{2}}{2 M_{A} 2 M_{B} 2 E_{1} 2 E_{2}} .
$$

Below we will use a natural generalization of this formula for particles with spin.

Our strategy is to use the standard Feynman rules with free initial and final particles to calculate the scattering amplitudes with the nonrelativistic initial particles. Then we convert the scattering amplitudes into effective potentials $V(\boldsymbol{r}, \boldsymbol{k})$, expand the integrand in Eq. (2) in spherical harmonics (with account for spin, if necessary), calculate the angular integrals analytically and finish with computing the remaining radial integrals numerically, using the wave functions obtained in [10].

Let us illustrate the logic of calculations still assuming that all particles in Fig. 1 are scalars. In this case the rescaled potential is just

$$
V(\boldsymbol{k}, \boldsymbol{q})=\frac{1}{M_{C}^{2}-(k-q)^{2}} .
$$

All external momenta are on mass shell and

$$
\begin{aligned}
M_{C}^{2}-(k-q)^{2} & =\left[M_{C}^{2}-\left(M_{A}-\sqrt{M_{1}^{2}+\boldsymbol{k}^{2}}\right)^{2}\right]+(\boldsymbol{k}-\boldsymbol{q})^{2} \\
& \equiv M_{*}^{2}(C)+(\boldsymbol{k}-\boldsymbol{q})^{2}
\end{aligned}
$$

and

$$
V(\boldsymbol{k}, \boldsymbol{q})=\frac{1}{M_{*}(C)^{2}+(\boldsymbol{k}-\boldsymbol{q})^{2}} .
$$

In this simple case the potential is a function only of $(\boldsymbol{k}-\boldsymbol{q})^{2}$ and its Fourier transform is just the Yukawa potential. Notice that its radius is determined not by the mass of the exchanged particle $M_{C}$ but by the effective $\operatorname{mass} M_{*}(C)=\sqrt{M_{C}^{2}-\left(M_{A}-\sqrt{M_{1}^{2}+\boldsymbol{k}^{2}}\right)^{2}}$.

\section{B. Tensor, spin, and isospin structure of decay potentials}

In the nonrelativistic approximation one-pion exchange in Fig. 1 generates a relatively long-range effective potential between $\Sigma_{c}$ and $\bar{D}^{*}$ that was used in [10] in discussion of the molecular pentaquark

$$
V(\boldsymbol{q})=-4 \frac{g_{\Sigma_{c}}^{A} g_{D^{*}}^{A}}{F_{\pi}^{2}}\left(\boldsymbol{t}_{1} \cdot \boldsymbol{t}_{2}\right) \frac{\left(\boldsymbol{s}^{(1)} \cdot \boldsymbol{q}\right)\left(\boldsymbol{s}^{(2)} \cdot \boldsymbol{q}\right)}{m_{\pi}^{2}+\boldsymbol{q}^{2}},
$$

where $g_{\Sigma_{c}}^{A}$ and $g_{D^{*}}^{A}$ are the axial charges of $\Sigma_{c}$ and $D^{*}$, respectively, and matrix elements of the spin and isospin operators $\boldsymbol{t}_{i}$ and $S_{i}$ should be calculated between the state vectors of the respective particles. In coordinate space the momentum-dependent factor turns into a superposition of a central and tensor potentials (we temporarily omit the coupling constants)

$$
\begin{aligned}
W_{i j}(\boldsymbol{r}) & =4 \int \frac{d^{3} q}{(2 \pi)^{3}} \frac{q_{i} q_{j}}{m_{\pi}^{2}+\boldsymbol{q}^{2}} e^{i \boldsymbol{q} \cdot \boldsymbol{r}} \\
& =V_{c}(r) \delta_{i j}+\left(3 n_{i} n_{j}-\delta_{i j}\right) V_{t}(r),
\end{aligned}
$$

where $n_{i}=r_{i} / r$ and

$$
\begin{aligned}
& V_{c}(r)=\frac{m^{2} e^{-m r}}{3 \pi r}, \\
& V_{t}(r)=\left[3+3 m r+(m r)^{2}\right] \frac{e^{-m r}}{3 \pi r^{3}} .
\end{aligned}
$$

There is also an additional term proportional to $\delta(\boldsymbol{r})$ on the right-hand side in Eq. (14). We omit it as unphysical in calculations of the bound state energies, because it arises from the distances where the one-pion exchange makes no sense due to finite sizes of all particles, see [10] for details. The spin and isospin matrices in Eq. (13) act in the space of spin and isospin states of the constituents. In [10] we used the potentials in Eqs. (13) and (15) together with the similar potentials that arise from $\sigma, \rho, \omega$ and $\eta$ exchanges to construct a loosely bound pentaquark state $P_{c}(4450)$. All potentials were regularized at small distances about $0.15 \mathrm{fm}$, for details of the regularization see Eq. $(31,32)$ in [10].

Decays of molecular pentaquarks without charm exchange can go via exchanges by a pion and other light mesons. We expect that the one-pion contribution, without account for exchanges by other mesons, gives a reasonable estimate of decay widths. Unlike the case of the binding potential, one-particle exchange decay amplitudes describe transitions from one pair of particles to another. After calculations pion exchange reduces to the potentials of the same type as in Eqs. (14) and (15), the only differences are that we use the nondiagonal axial charges (see also [24]), and substitute $m_{\pi} \rightarrow m_{*}(\pi)$ and $\boldsymbol{q}^{2} \rightarrow(\boldsymbol{k}-\boldsymbol{q})^{2}$, compare Eq. (11). Decays of the molecular and hadrocharmonium 
pentaquarks with exchange of charm go via $D$-meson and other heavy hadron exchanges. The respective effective potentials do not coincide with the ones in Eqs. (14) and (15), but still depend on spin, isospin and orbital momenta. This allows us to give a universal description of the strategy of further calculations. Consider, e.g., a molecular pentaquark decay. The bound state wave function of the molecular pentaquark [10] is a superpositions of the states $|l=0, S=3 / 2\rangle,|l=2, S=1 / 2\rangle$, and $|l=2, S=3 / 2\rangle$, where $l$ is the orbital momentum and $S$ is the total spin of the pentaquark. Each of the components of the molecular $\Sigma_{c} \bar{D}^{*}$ wave function is in its turn a superposition of one-particle spin-isospin states of the constituents. In terms of these spin-isospin states of the constituents the $\Sigma_{c} \bar{D}^{*}$ the wave function of the pentaquark in the state $\left|j=3 / 2, j_{3} ; t=1 / 2, t_{3}\right\rangle$ has the form

$$
\begin{aligned}
& \Psi_{2}^{\frac{3}{2}, j_{3}, \frac{1}{2}, t_{3}}(\boldsymbol{r})=\sum C_{S S_{3}, l m}^{\frac{3}{2} j_{3}} C_{\frac{1}{2} s_{3}^{(1)}, s_{3}^{(2)}}^{S S_{3}} C_{1 t_{3}, \frac{1}{2} t_{3}^{(2)}}^{\frac{1}{2}_{3}} R_{l S}(r) \\
& \times Y_{l m}(\boldsymbol{n}) \Sigma_{s_{3}^{(1)} t_{3}^{(1)}} \bar{D}_{s_{3}^{(2)} t_{3}^{(2)}}^{*},
\end{aligned}
$$

where $\Sigma_{s_{3}^{(1)} t_{3}^{(1)}}$ and $\bar{D}_{s_{3}^{(2)} t_{3}^{(2)}}$ are normalized to unity spinisospin states of $\Sigma_{c}$ and $\bar{D}^{*}$ with the spin projection $s_{3}^{(i)}$ and the isospin projection $t_{3}^{(i)}, j_{3}, t_{3}$ are the third components of the pentaquark spin and isospin, $Y_{l m}(\boldsymbol{n})$ are spherical harmonics, $C_{S S_{3}, l m}^{\frac{3}{2} j_{3}}, C_{\frac{1}{2} s_{3}^{(1)}, 1 s_{3}^{(2)}}^{S S_{3}}, C_{1 t_{3}^{2}, \frac{1}{2} t_{3}^{(2)}}^{\frac{1}{2} t_{3}}$ are the ClebschGordan coefficients, and $R_{l S}(r)$ are the radial wave functions in the states $|l, S\rangle$. Summation runs over spin and isospin projections of the constituents and includes also summation over three available $l, S$ combinations.

We consider a one-particle exchange scattering amplitude as an operator that acts on the initial wave function in Eq. (16) and transforms it in a superpositions of products of spin-isospin one-particle states of the final particles with the coefficients that are coordinate wave functions of their relative motion. Like in Eq. (16) these coordinate wave functions are themselves superpositions of products of radial wave functions and spherical harmonics. The final orbital momenta arise automatically by addition of orbital momenta of the initial wave function and of the interaction potential and do not coincide with the initial orbital momenta, only the total angular momentum is conserved in the general case. Next we project this wave function on the final plane wave, compare Eq. (3). We obtain a superposition of matrix elements of the potential $M(l, S \mid L)$ [compare Eq. (4)], with the coefficients that are spinisospin wave functions of the final particles. Unlike the expression in Eq. (4) the radial wave function $R_{l S}(r)$ carries now a second index $S$ because it depends on the total spin of the bound state. In addition the final angular momentum $L$ in the integral for $M(l, S \mid L)$ does not necessarily coincide with the initial angular momentum $l$ since the potential is in the general case a coordinate space (as well as spin and isospin) tensor. These matrix elements $M(l, S \mid L)$ are decay amplitudes of the initial state $|l, S\rangle$ into a final state with the total orbital momentum $L$ and spin-isospin quantum numbers of the coefficients.

To calculate the decay width in any channel we apply the operator arising from the respective one-particle exchange amplitude to the wave function Eq. (16) of the pentaquark with fixed quantum numbers. Then we obtain the decay amplitude as a superposition of matrix elements $M(l, S \mid L)$, square it, calculate the integrals over directions of the final momentum $\boldsymbol{k}$ and thus obtain the decay width. We will fill some technical gaps in this schematic discussion considering the decays below.

\section{DECAYS OF MOLECULAR PENTAQUARKS}

Let us recall the principal features of the molecular pentaquark scenario considered in [10]. Exotic pentaquarks in this picture are loosely bound states of hadrons with open charm located at rather large distances. One could expect that the interaction of the constituent hadrons in this case would be dominated by the long-range one-pion exchange and the pentaquark would resemble the deuteron, see, e.g., [25]. We considered this binding mechanism in [10] and came to the conclusion that the effective distances are not large enough to neglect exchanges by other light mesons, besides pions. The pion exchange in [10] was regularized to get rid of its unphysical too singular behavior at small distances, and exchanges by $\sigma, \rho, \omega$ and $\eta$ were also taken into account. Then we constructed the pentaquark $P_{c}(4450)$ as a loosely bound state of $\Sigma_{c}(2455)$ $\left(I\left(J^{P}\right)=1\left(1 / 2^{+}\right)\right)$and $\bar{D}^{*}(2010)\left(I\left(J^{P}\right)=1 / 2\left(1^{-}\right)\right)$with the binding energy only $15 \mathrm{MeV}$ and spin-parity $(3 / 2)^{-}$. This pentaquark arises when the regularization parameter $\Lambda=1300 \mathrm{MeV}$, with the root mean square radius $1.46 \mathrm{fm}$ and $D$-wave squared fraction about $4 \%$, see [10] for more details. An attempt to use the potential with the same parameters in order to construct $P_{c}(4380)$ as a loosely bound state of $\Sigma_{c}^{*}(2520)\left(I\left(J^{P}\right)=1\left(3 / 2^{+}\right)\right)$and $\bar{D}(1870)$ $\left(I\left(J^{P}\right)=1 / 2\left(0^{-}\right)\right)$with the binding energy $10 \mathrm{MeV}$ was not successful. The main reason is that the would be constituents $\Sigma_{c}^{*}$ and $\bar{D}$ do not interact via one-pion exchange since the three-pseudoscalar vertex $\pi D D$ is banned by parity, and exchanges by the other light mesons cannot provide the necessary binding. Therefore, if we insist that the LHCb $P_{c}(4380)$ pentaquark should be a loosely bound molecular state with a tiny binding energy its nature in this picture remains an open question.

Small binding energy and large size of the molecular pentaquark $P_{c}(4450)$ imply that the constituent hadrons are nonrelativistic and this bound state can be described in the potential approach. We constructed such molecular pentaquark in [10]. Let us consider its decays due to one-particle exchanges. 
TABLE I. Pentaquark $P_{c}(4450)$ decay widths in the molecular picture.

\begin{tabular}{lcccc}
\hline \hline Decay mode & $L^{\mathrm{a}}$ & $k^{\mathrm{b}}(\mathrm{MeV})$ & $m_{*}{ }^{\mathrm{c}}(\mathrm{MeV})$ & $\Gamma^{\mathrm{d}}(\mathrm{MeV})$ \\
\hline$P_{c} \rightarrow \Lambda_{c} \bar{D}$ & 2 & 798 & 136 & 6.8 \\
$P_{c} \rightarrow \Sigma_{c} \bar{D}$ & 2 & 529 & 128 & 1.4 \\
$P_{c} \rightarrow \Lambda_{c} \bar{D}^{*}$ & 0,2 & 579 & 101 & 13.3 \\
$P_{c} \rightarrow \Sigma_{c}^{*} \bar{D}$ & 0,2 & 360 & 107 & 0.2 \\
$P_{c} \rightarrow J / \psi N$ & 0 & 820 & 1421 & 0.03 \\
Total width & & & & 21.7 \\
\hline \hline
\end{tabular}

${ }^{\mathrm{a}}$ Lowest allowed orbital momentum.

${ }^{\mathrm{b}}$ Final momentum.

${ }^{\mathrm{c}}$ Effective exchanged mass.

${ }^{\mathrm{d}}$ Decay width.

\section{A. Decays into states with open charm}

There are four open channels for the $P_{c}(4450)$ pentaquark decays into states with open charm, see Table I. In the case of the molecular pentaquark there is no charm exchange in these decays and they can go via one-pion exchanges. As mentioned above, exchanges by heavier mesons are also allowed but we will account only for the contribution of the pion exchange.

\section{1. $P_{c} \rightarrow \Lambda_{c}+\bar{D}$ decay}

We start with the channel $P_{c} \rightarrow \Lambda_{c}+\bar{D}$. The initial pentaquark has spin-parity $3 / 2^{-}$and isospin $1 / 2$, the final $\Lambda_{c}$ carries spin-parity $1 / 2^{+}$and zero isospin, and the final $\bar{D}$ is a pseudoscalar with isospin $1 / 2$. The product of the internal parities of $\Lambda_{c}$ and $\bar{D}$ is negative, so the final state in the decay $P_{c}(4450) \rightarrow \Lambda_{c}+\bar{D}$ can have only even angular momenta. The final state with $L=0$ is banned by the angular momentum conservation, so the lowest allowed final orbital momentum is $L=2$. The final decay momentum is $k \approx 798 \mathrm{MeV}$, and both final particles are nonrelativistic with a reasonable accuracy, $E_{\Lambda} \approx 2421 \mathrm{MeV}$ and $\left(E_{\Lambda}-M_{\Lambda}\right) / M_{\Lambda} \approx 0.059$, and $E_{\bar{D}} \approx 2029 \mathrm{MeV}$ and $\left(E_{\bar{D}}-M_{\bar{D}}\right) / M_{\bar{D}} \approx 0.087$.

This decay is described by the diagram in Fig. 2. First we calculate the relativistic scattering amplitude in Fig. 3.

$\mathcal{A}(\boldsymbol{q}, \boldsymbol{k})=g_{\pi \Sigma_{c} \Lambda_{c}} g_{\pi D D^{*}} \bar{\Lambda}_{c}(\boldsymbol{k}) \gamma^{5} \frac{(k-q)_{\nu}}{m_{\pi}^{2}-(k-q)^{2}} \Sigma_{c}^{a} \bar{D}^{\dagger} \tau_{a} D^{* \nu}(\boldsymbol{q})$,

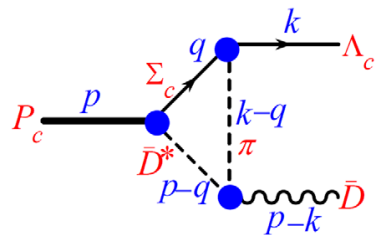

FIG. 2. Decay of molecular pentaquark $P_{c}(4450)$ into open charm states $\bar{D}+\Lambda_{c}$.

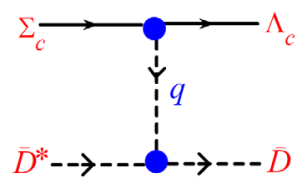

FIG. 3. Amplitude $\Sigma_{c}+\bar{D}^{*} \rightarrow \Lambda_{c}+\bar{D}$.

where $\bar{D}^{* \nu}(\boldsymbol{q})$ is a four-vector isospinor, $\bar{D}$ is an isospinor, $\Sigma_{c}^{a}$ is a spinor isovector, and $\Lambda_{c}(\boldsymbol{k})$ is a spinor. The coupling constants and interaction Lagrangians can be found in Table V and are discussed in Appendix A 1.

In the nonrelativistic approximation the denominator of the propagator reduces to $m_{*}^{2}(\pi)+(\boldsymbol{k}-\boldsymbol{q})^{2}$, and the interaction radius is determined by $m_{*}(\pi)=$ $\left\{m_{\pi}^{2}-\left[\left(M_{\Lambda_{c}}^{2}+\boldsymbol{k}^{2}\right)^{\frac{1}{2}}-M_{\Sigma_{c}}\right]^{2}\right\}^{\frac{1}{2}}=136 \mathrm{MeV}$. Using this approximation for the initial and final particles and omitting the coupling constants and certain square roots of masses [to be restored in the final expression for the decay width, compare Eq. (8)] we obtain the interaction potential that acts as an operator on the initial pentaquark wave function in Eq. (16)

$$
\left(\Lambda_{c}^{\dagger} \sigma^{i} \Sigma_{c}^{a}\right) W_{i k}(\boldsymbol{k}-\boldsymbol{q})\left(\bar{D}^{\dagger} \tau^{a} \bar{D}^{* k}\right)
$$

or in coordinate space

$$
\left(\Lambda_{c}^{\dagger} \sigma^{i} \sum_{c}^{a}\right) W_{i k}(\boldsymbol{r})\left(\bar{D}^{\dagger} \tau^{a} \bar{D}^{* k}\right),
$$

where $W_{i k}(\boldsymbol{r})$ is defined in Eq. (14) [now with $m \rightarrow m_{*}(\pi)$ ] and $\bar{D}, \bar{D}^{* k}, \Sigma_{c}^{a}, \Lambda_{c}$ are nonrelativistic spin-isospin states similar to the ones in Eq. (16).

It is convenient to represent $W_{i k}$ in terms of spherical harmonics ${ }^{3}$

$$
\begin{aligned}
W_{m_{1} m_{2}}(\boldsymbol{r})= & V_{c}(r)(-1)^{1-m_{1}} \delta_{m_{1},-m_{2}} \\
& -V_{t}(r) \sqrt{\frac{24 \pi}{5}} C_{1 m_{1}, 1 m_{2}}^{1, m_{1}+m_{2}} Y_{2,-m_{1}-m_{2}},
\end{aligned}
$$

where $V_{c}(r)$ and $V_{t}(t)$ are the regularized potentials in Eq. (15), see discussion of the regularization below Eq. (15) and in [10].

The transition operator in Eq. (19) should be applied to the initial wave function of the molecular pentaquark. We choose the initial pentaquark state with $j_{3}=3 / 2$ and $t_{3}=1 / 2$. The interaction operator in Eq. (19) transforms

$$
\begin{aligned}
& \begin{array}{l}
{ }^{3} \text { We use conventions for spherical harmonics from [26], in } \\
\text { particular }
\end{array} \\
& Y_{00}=\frac{1}{\sqrt{4 \pi}}, \quad Y_{20}=\sqrt{\frac{5}{16 \pi}}\left(1-3 n_{3}^{2}\right), \\
& Y_{2, \pm 1}= \pm \sqrt{\frac{15}{8 \pi}} n_{3}\left(n_{1} \pm i n_{2}\right), \quad Y_{2, \pm 2}=-\sqrt{\frac{15}{32 \pi}}\left(n_{1} \pm i n_{2}\right)^{2}
\end{aligned}
$$


it into the final wave function. After projection on the final plane wave and spatial integration we obtain the decay amplitude

$$
\begin{aligned}
\mathcal{M}_{i \rightarrow f}= & \frac{3}{\sqrt{5}}\left[M_{c}\left(2, \frac{1}{2} \mid 2\right)+M_{t}\left(0, \frac{3}{2} \mid 2\right)-M_{t}\left(2, \frac{3}{2} \mid 2\right)\right] \\
& \times Y_{21}(\boldsymbol{n}) \bar{D}^{0 \dagger} \Lambda_{c}^{\dagger}\left[\frac{1}{2}\right] \\
& -\frac{6}{\sqrt{5}}\left[M_{c}\left(2, \frac{1}{2} \mid 2\right)+M_{t}\left(0, \frac{3}{2} \mid 2\right)-M_{t}\left(2, \frac{3}{2} \mid 2\right)\right] \\
& \times Y_{22}(\boldsymbol{n}) \bar{D}^{0 \dagger} \Lambda_{c}^{\dagger}\left[-\frac{1}{2}\right],
\end{aligned}
$$

where $\Lambda_{c}^{\dagger}[ \pm 1 / 2]$ is the final $\Lambda_{c}$ with spin up or down, $\boldsymbol{n}=\boldsymbol{k} /|\boldsymbol{k}|$, and $M_{c, t}(l, S \mid L)$ are radial matrix elements of the potentials $V_{c, t}$ between the initial pentaquark state $|l, S\rangle$ and the final two-particle state with the orbital momentum $L=2$ similar to the ones in Eq. (4). We see that interaction in Eq. (19) generates only the transitions to the final states in $D$-wave. Next we calculate module square of the transition matrix element in Eq. (22), integrate over the directions of the final momentum, and sum over all allowed final states

$$
\begin{aligned}
& \underbrace{}_{f}\left|\mathcal{M}_{i \rightarrow f}\right|^{2} \\
& =9\left|M_{c}\left(2, \frac{1}{2} \mid 2\right)+M_{t}\left(0, \frac{3}{2} \mid 2\right)-M_{t}\left(2, \frac{3}{2} \mid 2\right)\right|^{2} .
\end{aligned}
$$

The decay width is calculated with a natural generalization of Eq. (9)

$$
\Gamma=g_{1}^{2} g_{2}^{2} \frac{4 k E_{1} E_{2}}{M_{P_{c}}} \frac{\mathbb{E}_{f}\left|\mathcal{M}_{i \rightarrow f}\right|^{2}}{\left(2 M_{1}\right)\left(2 M_{2}\right)\left(2 M_{A}\right)\left(2 M_{B}\right)},
$$

where we plug in $g_{1}=g_{\pi \Sigma_{c} \Lambda_{c}}, g_{2}=g_{\pi D D^{*}}, M_{A}=M_{\Sigma_{c}}$, $M_{B}=M_{D^{*}}, M_{1}=M_{\Lambda_{c}}, M_{2}=M_{D}, E_{1}=\sqrt{M_{\Lambda_{c}}^{2}+k^{2}}, E_{2}=$ $\sqrt{M_{D}^{2}+k^{2}}$, and sum of matrix elements squared from Eq. (23). We use Eq. (24) for calculations of all decay widths below.

After numerical calculations we obtain $\Gamma\left(P_{c} \rightarrow \Lambda_{c}+\bar{D}\right)=$ $6.8 \mathrm{MeV}$.

\section{Other open charm decays of molecular pentaquark}

Calculation of other three decays of the molecular pentaquark into states with an open charm

$P_{c} \rightarrow \Sigma_{c}+\bar{D}, \quad P_{c} \rightarrow \Lambda_{c}+\bar{D}^{*}, \quad P_{c} \rightarrow \Sigma_{c}^{*}+\bar{D}$,

is similar to the calculations above. All these decays go via the pion exchange, the final decay momenta are even

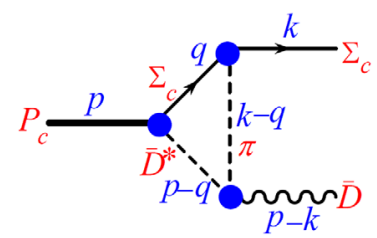

FIG. 4. Decay of molecular pentaquark $P_{c}(4450)$ into open charm states $\bar{D}+\Sigma_{c}$.

smaller than in the decay $P_{c} \rightarrow \Lambda_{c}+\bar{D}$, see Table I, and the decay products are nonrelativistic.

Decay $P_{c} \rightarrow \Sigma_{c}+\bar{D}$ requires almost no new calculations. Spin-parity of $\Sigma_{c}(2455)$ are the same as spin-parity of $\Lambda_{c}$ and like in the previous decay $L=2$ is the lowest allowed partial wave. The final momentum is $k \approx 529 \mathrm{MeV}$, and the final particles are again essentially nonrelativistic. Kinetic energy of the $D$-meson is about $4 \%$ of its mass, and kinetic energy of $\Sigma_{c}$ is about $2 \%$ of its mass.

The $P_{c} \rightarrow \Sigma_{c}+\bar{D}$ decay amplitude in Fig. 4 can be obtained from the decay amplitude $P_{c} \rightarrow \Lambda_{c}+\bar{D}$ in Fig. 2 . Only the isotopic structure of the $\pi \Sigma_{c} \Sigma_{c}$ vertex is different from the isotopic structure of the $\pi \Lambda_{c} \Sigma_{c}$ vertex, see the respective interaction Lagrangians in Table V. The isotopic factor factorizes in the decays amplitudes and the decay width of $P_{c} \rightarrow \Sigma_{c}+\bar{D}$ is equal to the decay width of $P_{c} \rightarrow \Lambda_{c}+\bar{D}$ times the ratio of the respective isotopic factors squared.

The isospinor isotopic factor in the molecular pentaquark wave function is $\Psi_{\alpha}^{\text {iso }}=(1 / \sqrt{3}) \Sigma_{c}^{a}\left(\tau^{a}\right)_{\alpha \beta} \bar{D}_{\beta}^{*}$. In the case of $P_{c} \rightarrow \Lambda_{c}+\bar{D}$ decay we apply to this wave function the isotopic factor $\tau^{a}$ in the transition operator in Eq. (19) and obtain the final isotopic function

$\Psi_{\mathrm{fin}}^{\mathrm{iso}, \alpha}\left(\bar{D}+\Lambda_{c}\right)=\frac{1}{\sqrt{3}}\left(\tau_{a} \tau_{a}\right)_{\beta}^{\alpha} \bar{D}^{\beta} \Lambda_{c}=\sqrt{3} \delta_{\beta}^{\alpha} \bar{D}^{\beta} \Lambda_{c}$.

In the case of the $P_{c} \rightarrow \Sigma_{c}+\bar{D}$ decay the isotopic factor in the transition operator in the diagram in Fig. 4 is $\tau_{a} \epsilon_{a b c}$ and then the final isotopic wave function is

$$
\begin{aligned}
\Psi_{\mathrm{fin}}^{\mathrm{iso}, \alpha}\left(\bar{D}+\Sigma_{c}\right) & =\frac{1}{\sqrt{3}}\left(\tau^{b} \tau^{c}\right)_{\beta}^{\alpha} \varepsilon_{a b c} \bar{D}^{\beta} \Sigma_{c}^{a} \\
& =\frac{2 i}{\sqrt{3}}\left(\tau^{a}\right)_{\beta}^{\alpha} \bar{D}^{\beta} \Sigma_{c}^{a} .
\end{aligned}
$$

Squaring the isotopic factors in the scattering amplitudes and summing over all allowed final isotopic states we obtain the isotopic factor contributions to the decay width in both cases

$$
\begin{aligned}
& \Phi^{\mathrm{iso}}\left(P_{c} \rightarrow \Lambda_{c}+\bar{D}\right)=3 \\
& \Phi^{\mathrm{iso}}\left(P_{c} \rightarrow \Sigma_{c}+\bar{D}\right)=\frac{4}{3}\left(\tau^{a} \tau^{a}\right)_{\alpha}^{\alpha}=4 .
\end{aligned}
$$


Spin and orbital structure of the matrix elements is identical for both decays. Hence, the sum of matrix elements squared for the decay $P_{c} \rightarrow \Sigma_{c}+\bar{D}$ is $4 / 3$ times larger than the sum of matrix elements squared for the decay $P_{c} \rightarrow \Lambda_{c}+\bar{D}$, and [compare Eq. (23)]

$$
\begin{aligned}
& y_{f}\left|M_{i \rightarrow f}\right|^{2} \\
& =12\left|M_{c}\left(2, \frac{1}{2} \mid 2\right)+M_{t}\left(0, \frac{3}{2} \mid 2\right)-M_{t}\left(2, \frac{3}{2} \mid 2\right)\right|^{2}
\end{aligned}
$$

for $P_{c} \rightarrow \Sigma_{c}+\bar{D}$.

Calculating the width according to Eq. (24) we obtain $\Gamma\left(P_{c} \rightarrow \Sigma_{c}+\bar{D}\right)=1.4 \mathrm{MeV}$.

The $P_{c} \rightarrow \Lambda_{c}+\bar{D}^{*}$ decay goes via the one-pion exchange diagram in Fig. 5. The $D^{*} D^{*} \pi$ interaction

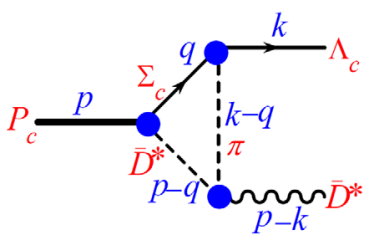

FIG. 5. Decay of molecular pentaquark $P_{c}(4450)$ into open charm states $\bar{D}^{*}+\Lambda_{c}$.

Lagrangian and coupling constant are in Table V. Let us notice that both interaction constants in this decay are found from the experimental data on decays, see discussion in Appendix A 1.

We go through by now the standard steps and obtain a rather cumbersome sum of matrix elements squared for this decay

$$
\begin{aligned}
y_{f}\left|M_{i \rightarrow f}\right|^{2}= & \frac{3}{5}\left|M_{c}\left(2, \frac{3}{2} \mid 2\right)+2 M_{t}\left(0, \frac{3}{2} \mid 2\right)-M_{t}\left(2, \frac{1}{2} \mid 2\right)\right|^{2}+3\left|M_{c}\left(0, \frac{3}{2} \mid 0\right)+M_{t}\left(2, \frac{1}{2} \mid 0\right)+2 M_{t}\left(2, \frac{3}{2} \mid 0\right)\right|^{2} \\
& +\frac{1}{5}\left|2 M_{c}\left(2, \frac{1}{2} \mid 2\right)+2 M_{c}\left(2, \frac{3}{2} \mid 2\right)+3 M_{t}\left(0, \frac{3}{2} \mid 2\right)-2 M_{t}\left(2, \frac{1}{2} \mid 2\right)+M_{t}\left(2, \frac{3}{2} \mid 2\right)\right|^{2} \\
& +\frac{6}{5}\left|2 M_{c}\left(2, \frac{1}{2} \mid 2\right)-M_{c}\left(2, \frac{3}{2} \mid 2\right)-3 M_{t}\left(0, \frac{3}{2} \mid 2\right)+M_{t}\left(2, \frac{1}{2} \mid 2\right)+M_{t}\left(2, \frac{3}{2} \mid 2\right)\right|^{2} \\
& +\frac{2}{5}\left|4 M_{c}\left(2, \frac{1}{2} \mid 2\right)+M_{c}\left(2, \frac{3}{2} \mid 2\right)-M_{t}\left(2, \frac{1}{2} \mid 2\right)+2 M_{t}\left(2, \frac{3}{2} \mid 2\right)\right|^{2} .
\end{aligned}
$$

This sum is dominated by the second term that describes transitions between the states with zero orbital momentum. We substitute this sum in Eq. (24) and obtain $\Gamma\left(P_{c} \rightarrow \Lambda_{c}+\bar{D}^{*}\right)=13.3 \mathrm{MeV}$.

The $P_{c} \rightarrow \Sigma_{c}^{*}+\bar{D}$ decay goes via the one-pion exchange diagram in Fig. 6. The $\pi \Sigma_{c}^{*} \Sigma_{c}$ interaction Lagrangian and coupling constant are in Table V. After calculations we obtain the sum of matrix elements squared

$$
\begin{aligned}
& \sum_{f}\left|\mathcal{M}_{i \rightarrow f}\right|^{2} \\
& =2\left|M_{c}\left(2, \frac{3}{2} \mid 2\right)-M_{t}\left(0, \frac{3}{2} \mid 2\right)-M_{t}\left(2, \frac{1}{2} \mid 2\right)\right|^{2} \\
& \quad+2\left|M_{c}\left(0, \frac{3}{2} \mid 0\right)+M_{t}\left(2, \frac{1}{2} \mid 0\right)-M_{t}\left(2, \frac{3}{2} \mid 2\right)\right|^{2},
\end{aligned}
$$

substitute it in Eq. (24) and calculate the width $\Gamma\left(P_{c} \rightarrow \Sigma_{c}^{*}+\bar{D}\right)=0.2 \mathrm{MeV}$.

\section{B. Decays into states with hidden charm}

The $P_{c}(4450) \rightarrow J / \psi+N$ decay is the only one kinematically allowed two-particle decay of the pentaquark into states with hidden charm. This decay goes via diagrams with exchange by a charmed meson or baryon in $t$-channel, e.g., $D, D^{*}, \Sigma_{c}$, etc. We will account only for the contribution of the diagram in Fig. 7 with the exchange by the lightest charmed particle, the pseudoscalar $D$, that we expect to provide a reasonable estimate of the total decay width. The product of internal parities of $J / \psi$ and $N$ is negative, so decay $P_{c}(4450) \rightarrow J / \psi+N$ goes with the lowest orbital momenta $L=0,2$. The decay momentum $k=820 \mathrm{MeV}$ in this decay is comparable with the nucleon mass and one cannot use the nonrelativistic approximation for the final nucleon.

As with the pion exchanges above, we start with calculation of the relativistic scattering amplitude in Fig. 8.

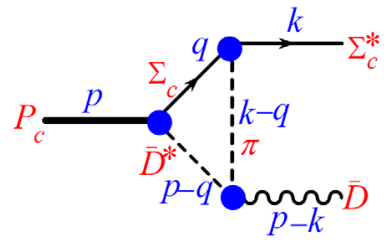

FIG. 6. Decay of molecular pentaquark $P_{c}(4450)$ into open charm states $\bar{D}+\Sigma_{c}^{*}$. 


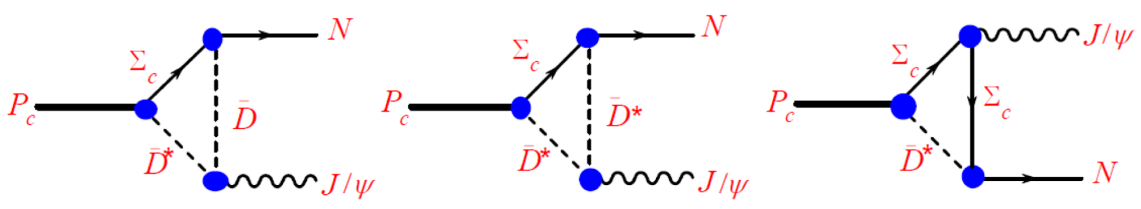

FIG. 7. Decays of the molecular pentaquark $P_{c}(4450)$ into hidden charm states $J / \psi+N$.

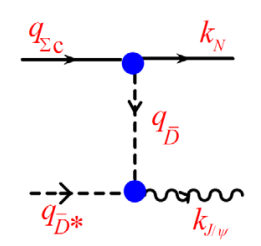

FIG. 8. Amplitude $\Sigma_{c}+\bar{D}^{*} \rightarrow N+J / \psi$.

$$
\begin{aligned}
\mathcal{A}(\boldsymbol{q}, \boldsymbol{k})= & g_{\Sigma_{c} D N} g_{J / \psi D D^{*}} \epsilon^{* \nu} \bar{N}(k) \gamma^{5} \tau^{a} \\
& \times \frac{1}{M_{D}^{2}-q_{D}^{2}} \epsilon^{\mu \nu \alpha \beta} k_{\mu}^{J / \psi}\left(q_{D}-q_{\bar{D}^{*}}\right)_{\beta} \Sigma_{c}^{a} \bar{D}_{\alpha}^{*},
\end{aligned}
$$

where $\bar{D}_{\alpha}^{*}(\boldsymbol{q})$ is a four-vector isospinor, $\Sigma^{a}$ is a spinor isovector, $N(\boldsymbol{k})$ is a spinor isospinor, and $\epsilon^{\nu}$ is the polarization vector of the final $J / \psi$. The coupling constants and interaction Lagrangians can be found in Tables VI and VII, and are discussed in Appendices A 2 and A 3.

Next we would like to make a nonrelativistic expansion in the initial momentum $\boldsymbol{q}$. The denominator of the propagator in Eq. (32) reduces to $M_{*}^{2}(D)+(\boldsymbol{k}-\boldsymbol{q})^{2}$ and the range of the effective potential is determined by $M_{*}(D)=$ $\left[M_{D}^{2}-\left(M_{\Sigma_{c}}-E_{N}\right)^{2}\right]^{\frac{1}{2}}=1421 \mathrm{MeV} \quad\left(E_{N}=\sqrt{M_{N}^{2}+\boldsymbol{k}^{2}}\right)$. This effective potential acts at shorter distances than in the case of the molecular pentaquark decays into states with open charm. The zero component of the transferred momentum $M_{\Sigma_{c}}-\sqrt{M_{N}^{2}+k^{2}}=1208 \mathrm{MeV}$ is also large. Hence, we cannot neglect the decay momentum and zero component of the transferred momentum in the nonrelativistic limit. As a result the coordinatedependent term $W_{i k}(\boldsymbol{r})$ in the transition operator

$$
\bar{N}^{\dagger} \sigma_{i} \Sigma_{c}^{a} \tau^{a} \bar{D}_{l}^{*} \epsilon_{m}^{*} \varepsilon_{k l m} W_{i k}
$$

is more complicated than the similar term $W_{i k}(\boldsymbol{r})$ from Eq. (14) in a fully nonrelativistic case in Eq. (19). In the case at hand

$$
\begin{aligned}
W_{i k}(\boldsymbol{r})= & \delta_{i k} V_{c}(r)+\left(3 n_{i} n_{k}-\delta_{i k}\right) V_{t}(r) \\
& +\left[i\left(a_{1} k_{i} \partial_{k}+a_{2} k_{k} \partial_{i}\right)+b k_{i} k_{k}\right] \frac{3 V_{c}(r)}{M_{*}^{2}(D)}
\end{aligned}
$$

The derivatives originate from the linear in the relative momentum $\boldsymbol{q}$ terms $q_{i} k_{k}$ in the numerator of the momentum space expressions. Due to these derivatives a new potential

$$
V_{d}(r)=\frac{\partial}{\partial r}\left[\frac{3 V_{c}(r)}{M_{*}(D)}\right]
$$

arises in $W_{i k}(\boldsymbol{r})$ in Eq. (34) besides the potentials $V_{c}$ and $V_{t}$ from Eq. (15) $\left(M_{*}(D)\right.$ plays the role of the mass parameter in all three potentials). We also keep the last bilinear in the final momentum $k_{i} k_{k}$ term in the square brackets in Eq. (34) that cannot be legitimately omitted when the final momentum is large. All these new terms are missing in the nonrelativistic decays with exchange by an almost massless pseudoGoldstone pion, because its interaction vertex is always proportional to its momentum. But nothing bans such interaction terms for a heavy $\bar{D}$.

The coefficients in Eq. (34) are functions of masses and the final momentum

$a_{1}=1-\frac{2 M_{\Sigma_{c}}}{M_{N}+E_{N}}, \quad a_{2}=\frac{M_{\Sigma_{c}}-E_{N}}{E_{J / \psi}}, \quad b=-a_{1} a_{2}$,

where $E_{J / \psi}=\sqrt{M_{J / \psi}+k^{2}}$ is the energy of the produced $J / \psi$. Notice that these coefficients would be zero if masses of the constituent $\Sigma_{c}$ and the produced nucleon were close.

Further calculations go almost as in the case of the nonrelativistic decays above. A new element is connected with the scalar products like $\boldsymbol{k} \cdot \boldsymbol{n}(\boldsymbol{n}=\boldsymbol{r} / \boldsymbol{r})$ that arise after differentiation in Eq. (34). We write them in terms of spherical harmonics $\boldsymbol{k} \cdot \boldsymbol{n}=-i \sqrt{4 \pi / 3} \sum_{m} k^{(-m)} Y_{1 m}$, where $k^{(-m)}$ are spherical components of $\boldsymbol{k}$. After application of the transition operator the final wave function contains products of different spherical harmonics that depend on $\boldsymbol{r} / r$ and we use the Clebsch-Gordan coefficients to obtain terms linear in spherical harmonics, integrate over angles with the outgoing plane wave and obtain typical terms $j_{L}(k r) Y_{L M}(\boldsymbol{k} / k)$. Unlike the decays considered above, now such terms are multiplied by linear in the spherical components of $\boldsymbol{k}$ factors. We calculate the radial integrals, project each of the products of spherical harmonics of $\boldsymbol{k} / \boldsymbol{k}$ on a single spherical harmonic $Y_{L^{\prime} M}(\boldsymbol{k} / k)$, square the obtained sums and integrate over directions of $\boldsymbol{k}$. Notice that this calculation leads to the decay products with a final orbital momentum $L^{\prime} \neq L$ in $M(l, S \mid L)$ ( $L$ is the label of the spherical Bessel function in the respective radial integral). The expression for the sum of matrix elements squared turns out to be rather cumbersome. The dominant contribution to this sum is supplied by the transitions from the 
component of the initial bound state wave function with $l=0, S=3 / 2$ that has the form

$$
\begin{aligned}
\sum_{f}\left|\mathcal{M}_{i \rightarrow f}\right|^{2}= & 3\left(1+\frac{2 b k^{2}}{M_{*}^{2}(D)}+\frac{6 b^{2} k^{4}}{M_{*}^{4}(D)}\right)\left|M_{c}\left(0, \frac{3}{2} \mid 0\right)\right|^{2} \\
& +15\left|M_{t}\left(0, \frac{3}{2} \mid 2\right)\right|^{2} \\
& +\frac{30 b k^{2}}{M_{*}^{2}(D)} M_{t}\left(0, \frac{3}{2} \mid 2\right) M_{c}\left(0, \frac{3}{2} \mid 0\right) \\
& +\frac{2\left(a_{1}+a_{2}\right)^{2} k^{2}}{M_{*}^{2}(D)}\left|M_{d}\left(0, \frac{3}{2} \mid 1\right)\right|^{2}
\end{aligned}
$$

where we introduced matrix element of a new type

$$
M_{d}(l, S \mid L)=\int_{0}^{\infty} d r r^{2} R_{l S}(r) V_{d}(r) j_{L}(k r)
$$

that arises only for the odd values of $L$. The potential $V_{d}(r)$ in this integral is regularized in the same way as the potentials $V_{c}(r)$ and $V_{t}(r)$ in Eq. (15).

The final nucleon is relativistic in this decay and the general formula for the width in Eq. (24) changes

$$
\begin{aligned}
\Gamma= & g_{D \Sigma_{c} N}^{2} g_{J / \psi D D^{*}}^{2} \frac{4 k E_{N} E_{J / \psi}}{M_{P_{c}}} \\
& \times \frac{E_{J / \psi}^{2}}{\left(2 M_{D^{*}}\right)\left(2 M_{\Sigma_{c}}\right)\left(2 E_{J / \psi}\right)\left(2 E_{N}\right)} \frac{E_{N}+M_{N}}{2 M_{\Sigma_{c}}} \searrow_{f}\left|\mathcal{M}_{i \rightarrow f}\right|^{2} .
\end{aligned}
$$

After numerical calculations we obtain decay width of the molecular pentaquark into states with hidden charm $\Gamma\left(P_{c}(4450) \rightarrow N+J / \psi\right)=0.03 \mathrm{MeV}$. Account for relativity of the final nucleon significantly affects this result, the width decreases by $61 \%$ without the relativistic corrections. The suppression of the decay into hidden charm states is somewhat stronger that the one we could expect from the estimates of the matrix elements discussed in the next section. This additional suppression is due to the small magnitude of the coupling constant $g_{\Sigma_{c} N D}$, see Table VI and discussion in Appendix A 2. Let us emphasize that a rather strong suppression due to smallness of the matrix elements would survive even a significant increase of the coupling constant.

\section{Comparison of molecular pentaquark decays into states with hidden and open charm}

The results collected in Table I demonstrate that the decay into states with hidden charm is suppressed in comparison with the decays into states with open charm in the molecular picture. As already mentioned in the Introduction this happens because an exchange by a heavy charmed particle is required in decays to the hidden charm states. Let us recap the arguments given in the Introduction. We argued that in order to decay into hidden charm state the constituents in the molecular picture should come to a small distance $\sim 1 / m_{c}$. This is a tiny scale in comparison with the scale of the wave function $\sim 1 / \kappa \gg 1 / m_{c}$ and therefore this width is proportional to $\int d^{3} r|\psi(\boldsymbol{r})|^{2} \sim|\psi(0)|^{2} / m_{c}^{3} \sim\left(\kappa / m_{c}\right)^{3}$. For molecular pentaquark $\kappa=\sqrt{2 \mu \epsilon} \approx 182 \mathrm{MeV}$ and $\left(\kappa / m_{c}\right)^{3} \sim 3 \times 10^{-3}$. As we will show below this estimate is too naive and the characteristic distance in molecular decays into states with hidden charm is determined not by $m_{c}$ but by the mass of a heavy exchanged particle, with the effective mass $M_{*}$ that grows only as $\sqrt{m_{c}}$ with $m_{c}$.

Let us try to improve the naive estimate of molecular decays into states with hidden charm. Recall that the decay amplitudes are sums of the overlap integrals similar to the ones in Eqs. (4) and (38)

$$
M(l, S \mid L)_{c, d, t}=\int_{0}^{\infty} d r r^{2} R_{l S}(r) V_{c, d, t}(r) j_{L}(k r)
$$

where the potentials are defined in Eqs. (15) and (38). We collected results of the numerical calculations of matrix elements $M(l, S \mid L)$ for a typical decay without charm exchange in Table III and with charm exchange in Table II, respectively.

In decays with charm exchange the effective mass $M_{*}$ is much larger than the decay momentum $k$ and the scale of the wave function $\kappa, M_{*} \gg k>\kappa$, see Table I. Then

\begin{tabular}{|c|c|c|c|c|c|c|c|c|c|}
\hline & $M\left(0, \frac{3}{2} \mid 0\right)$ & $M\left(2, \frac{1}{2} \mid 0\right)$ & $M\left(2, \frac{3}{2} \mid 0\right)$ & $M\left(0, \frac{3}{2} \mid 1\right)$ & $M\left(2, \frac{1}{2} \mid 1\right)$ & $M\left(2, \frac{3}{2} \mid 1\right)$ & $M\left(0, \frac{3}{2} \mid 2\right)$ & $M\left(2, \frac{1}{2} \mid 2\right)$ & $M\left(2, \frac{3}{2} \mid 2\right)$ \\
\hline$V_{c}$ & 0.0232835 & $1.48 \times 10^{-3}$ & $-3.72 \times 10^{-3}$ & & & & $-4.33 \times 10^{-3}$ & $2.47 \times 10^{-4}$ & $-6.44 \times 10^{-4}$ \\
\hline$V_{t}$ & & $-7.10 \times 10^{-3}$ & $-1.74 \times 10^{-2}$ & & & & $-1.37 \times 10^{-2}$ & $6.37 \times 10^{-4}$ & $-1.64 \times 10^{-3}$ \\
\hline$V_{d}$ & & & & $7.76 \times 10^{-2}$ & $-3.11 \times 10^{-3}$ & $7.96 \times 10^{-3}$ & & & \\
\hline
\end{tabular}

$$
\begin{aligned}
M_{c, d, t}(l, S \mid L) & \sim \int_{0}^{\frac{1}{M_{*}}} d r r^{2}(\kappa r)^{l}(k r)^{L} V_{c, d, t}(r) \\
& \sim\left(\frac{\kappa}{M_{*}}\right)^{l}\left(\frac{k}{M_{*}}\right)^{L} \frac{V_{c, d, t}\left(\frac{1}{M_{*}}\right)}{M_{*}^{3}} .
\end{aligned}
$$

The sum $l+L \geq 2$ in the integrals with the tensor potential and the overlap matrix element is at most $M_{t} \sim\left(k / M_{*}\right)^{2}$ at $l=0$ and $L=2$. In the integral with the potential $V_{d}(r) L$ is always odd, and this integral is at most $M_{d} \sim k / M_{*}$ at

TABLE II. Molecular pentaquark decay $P_{c} \rightarrow J / \psi+N$ : matrix elements. 
TABLE III. Molecular pentaquark decay $P_{c} \rightarrow \Lambda_{c}+\bar{D}^{*}$ : Matrix elements.

\begin{tabular}{ccccccc}
\hline \hline & $M\left(0, \frac{3}{2} \mid 0\right)$ & $M\left(2, \frac{1}{2} \mid 0\right)$ & $M\left(2, \frac{3}{2} \mid 0\right)$ & $M\left(0, \frac{3}{2} \mid 2\right)$ & $M\left(2, \frac{1}{2} \mid 2\right)$ & $M\left(2, \frac{3}{2} \mid 2\right)$ \\
\hline$V_{c}$ & $-1.95 \times 10^{-3}$ & $1.09 \times 10^{-4}$ & $-2.86 \times 10^{-4}$ & $-8.90 \times 10^{-4}$ & $6.97 \times 10^{-5}$ & $-1.86 \times 10^{-4}$ \\
$V_{t}$ & & $1.36 \times 10^{-2}$ & $-3.43 \times 10^{-2}$ & $-2.96 \times 10^{-2}$ & $2.00 \times 10^{-3}$ & $-5.30 \times 10^{-3}$ \\
\hline \hline
\end{tabular}

$l=0$ and $L=1$. It enters the decay amplitude with an additional factor $k / M_{*}$ and as a result contributes to the decay amplitude at most $\left(k / M_{*}\right)^{2}$, exactly like the tensor potential. Finally, naively the contribution of the central potential $V_{c}$ to the integral in Eq. (40) at $l=L=0$ seems to be independent of $M_{*}$ when $M_{*}$ increases. This contradicts the well-grounded physical expectations that exchange by a very massive particle should supply negligible contribute to the decay width. It is not hard to figure out what happened. Calculating the Fourier transform in Eq. (14) we have thrown away the $\delta$-function term as unphysical in the case of exchange by a light pion. However, the calculation above shows that for a heavy exchange this $\delta$-function is necessary to restore the proper dependence of the $l=L=0$ decay matrix element on mass of the exchanged particle. It is easy to see that restoration of $\delta$-function reduces to substitution $M_{c}(0, S \mid 0) \rightarrow M_{c}(0, S \mid 0)-R_{0 S}(0) /(12 \pi)$. We made this subtraction in calculations of all molecular and hadrocharmonium decays with charm exchange. The subtracted matrix elements are at most $\left(k / M_{*}\right)^{2}$ and we conclude that effectively all matrix elements in Eq. (40) decrease with $M_{*}$ as $\left(k / M_{*}\right)^{2}$ or faster.

Molecular decays into open charm states go via exchange by the light pion, only the potentials $V_{c, t}$ give contribution to these decays, and $m_{*} \sim m_{\pi}$. Numerically, in this case $m_{*} \sim \kappa \ll k$ Then integration in Eq. (40) goes up to $r \sim 1 / k \ll 1 / \kappa \sim 1 / m_{*}$ and

$$
M(l, S \mid L)_{c, t} \sim \int_{0}^{\frac{1}{k}} d r r^{2}(\kappa r)^{l} V_{c, t}(r) j_{L}(k r) .
$$

In this region the matrix element of the scalar potential $M(l, S \mid L)_{c} \sim(\kappa / k)^{l}\left(m_{*} / k\right)^{2}$ is suppressed in comparison with the matrix element of the tensor potential $M(l, S \mid L)_{t} \sim$ $(\kappa / k)^{l} \sim\left(m_{*} / k\right)^{l}$ by the factor $\left(k / m_{*}\right)^{2} \sim 15-30$.

Now we can estimate ratio $R$ of matrix elements for decay into states with hidden and open charm

$$
R \sim\left(\frac{k_{\mathrm{hid}}}{M_{*}}\right)^{L} /\left(\frac{m_{*}}{k_{\mathrm{open}}}\right)^{l}
$$

where $k_{\text {open }}$ and $k_{\text {hid }}$ are decay momenta in the hidden and open charm decays, respectively, and $M_{*}=M_{*}(D)$. We compare matrix elements for hidden charm decays with the tensor matrix elements in open charm decays since scalar matrix elements in open charm decays are suppressed. Numerically for decays in Tables II and III
$R \sim 0.4^{l} \times 0.5^{L} \sim 0.1-0.2$. Respectively, we expect that the hidden charm decays of the molecular pentaquark should be suppressed by a factor 0.01-0.04, what is compatible with the results in Table I. This suppression is weaker than the naive suppression factor $\left(\kappa / m_{c}\right)^{3} \sim 10^{-6}$ discussed above.

\section{HADROCHARMONIUM DECAYS}

\section{A. Decays into states with hidden charm}

In the hadrocharmonium picture the $\mathrm{LHCb}$ pentaquark $P_{c}(4450)$ is interpreted as a bound state of $\psi^{\prime}$ and the nucleon $[9,10]$ (see also [11]). It is described by a nonrelativistic wave function that is a product of the $S$-wave coordinate wave function and the spin $3 / 2$ and isospin $1 / 2$ factor. The partial decay width of the hadrocharmonium pentaquark $\Gamma\left(P_{c}(4450) \rightarrow J / \psi+N\right) \approx 11 \mathrm{MeV}$ was calculated in $[9,10]$. As mentioned above this is the only one kinematically allowed two-particle pentaquark decay channel into states without open charm.

\section{B. Decays into states with open charm}

Hadrocharmonium decays into states with open charm go via exchange by heavy hadrons. As in the molecular decays we will take into account only exchanges by the lightest particle with open charm, namely by $D$-meson. We expect that the respective partial widths are reasonably well approximated by this exchange. The inverse size $\kappa=$ $\sqrt{2 \mu \epsilon}=506 \mathrm{MeV}$ of the hadrocharmonium pentaquark wave function is determined by its binding energy $\epsilon=$ $178 \mathrm{MeV}$ and reduced mass $\mu=720 \mathrm{MeV}$. Recall that in the case of the molecular pentaquark we obtained $\kappa=182 \mathrm{MeV}$. Hence, the hadrocharmonium wave function is less extended and is larger at the origin than the molecular one. This favors decays with exchange of charm and one can expect that the hadrocharmonium decays into states with open charm have larger partial widths than the molecular pentaquark decay into $J / \psi N$. It is harder to anticipate relative magnitude of partial decay widths into states with open charm in the hadrocharmonium and molecular pictures. On the one hand larger at the origin and less extended hadrocharmonium wave function could probably enhance decay rates into the four channels with open charm. On the other hand the effective masses of the exchanged particles in these decays are much higher than in the case of the molecular pentaquark (compare Tables I and IV), what works in the opposite direction. Only calculations will show which effect is more pronounced. 


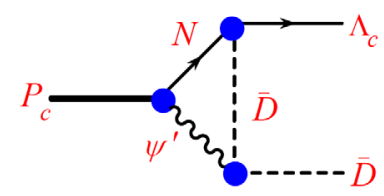

FIG. 9. Decay of hadrocharmonium pentaquark $P_{c}(4450)$ into states with open charm $\Lambda_{c}+\bar{D}$.

$$
\text { 1. } P_{c} \rightarrow \Lambda_{c}+\bar{D}
$$

Consider first the hadrocharmonium decay $P_{c} \rightarrow \Lambda_{c}+\bar{D}$. Kinematics of this decay was already discussed above. This decay can go via exchange by the $D$-meson and heavier particles with open charm. As already explained we calculate the partial decay width due to the diagram with the pseudoscalar $D$ exchange in Fig. 9 and expect that this exchange provides a reasonable estimate of the total partial decay width into $\Lambda_{c}$ and $\bar{D}$.

As usual we first calculate the relativistic scattering amplitude $N+\psi^{\prime} \rightarrow \Lambda_{c}+\bar{D}$ in Fig. 10 (momenta are labeled as in the figure)

$$
\begin{aligned}
\mathcal{A}(\boldsymbol{q}, \boldsymbol{k})= & g_{\Lambda_{c} D N} g_{\psi^{\prime} D D} \bar{\Lambda}_{c}\left(\boldsymbol{k}_{\Lambda_{c}}\right) \gamma^{5} \bar{D}^{\dagger} \\
& \times \frac{1}{M_{D}^{2}-q_{e D}^{2}}\left(q_{\bar{D}}+k_{\bar{D}^{*}}\right)^{\alpha} \Phi_{\alpha} N,
\end{aligned}
$$

where $\Phi_{\alpha}(\boldsymbol{q})$ is a four-vector that describes initial $\psi^{\prime}, N$ is a spinor isospinor, $D$ is an isospinor, and $\Lambda_{c}\left(\boldsymbol{k}_{\Lambda}\right)$ is a spinor. The isospin indices are contracted along the virtual $\bar{D}$ line. The coupling constants and interaction Lagrangians are collected in Tables VI and VII and discussed in Appendices A 2 and A 3.

In the nonrelativistic expansion in the initial momentum $\boldsymbol{q}$ the denominator of the propagator in Eq. (44) reduces to $M_{*}^{2}(D)+(\boldsymbol{k}-\boldsymbol{q})^{2}$ and the range of the effective potential is determined by $M_{*}(D)=\left[M_{D}^{2}-\left(E_{\Lambda_{c}}-M_{N}\right)^{2}\right]^{\frac{1}{2}} \approx 1133 \mathrm{MeV}$ $\left(E_{\Lambda_{c}}=\left(M_{\Lambda_{c}}^{2}+\boldsymbol{k}^{2}\right)^{\frac{1}{2}}\right)$. The relativistic amplitude in the nonrelativistic limit reduces to the transition operator

$$
\left(\Lambda_{c}^{\dagger} \sigma^{i} N^{a}\right) W_{i k}(\boldsymbol{r})\left(D^{\dagger a} \psi_{k}^{\prime}\right)
$$

where $W_{i k}(\boldsymbol{r})$ has the same form as in Eq. (34) with the natural kinematic substitutions and

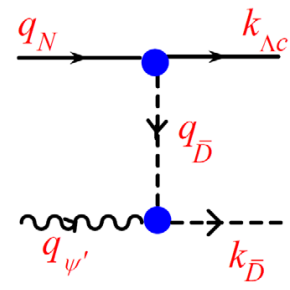

FIG. 10. Amplitude $N+\psi^{\prime} \rightarrow \Lambda_{c}+\bar{D}$. $a_{1}=1-\frac{2 M_{N}}{E_{\Lambda_{c}}+M_{\Lambda_{c}}}, \quad a_{2}=-1, \quad b=-a_{1} a_{2}$.

We preserved the external momentum $k$ in the transition operator. Next we apply the transition operator to the initial wave function [compare Eq. (22)] and calculate the sum of matrix elements squared of the transition amplitude [compare Eq. (23)]

$$
\begin{aligned}
\sum_{f}\left|M_{i \rightarrow f}\right|^{2} \\
=3\left|M_{t}\left(0, \frac{3}{2} \mid 2\right)\right|^{2}+\frac{\left(a_{1}+a_{2}\right)^{2} k^{2}}{3 M_{*}^{2}(D)}\left|M_{d}\left(0, \frac{3}{2} \mid 1\right)\right|^{2} \\
\quad+\frac{3 b^{2} k^{4}}{M_{*}^{4}(D)}\left|M_{c}\left(0, \frac{3}{2} \mid 0\right)\right|^{2} \\
\quad+\frac{6 b k^{2}}{M_{*}^{2}(D)} M_{c}\left(0, \frac{3}{2} \mid 0\right) M_{t}\left(0, \frac{3}{2} \mid 2\right) .
\end{aligned}
$$

The partial decay width is [compare Eq. (39)]

$$
\begin{aligned}
\Gamma\left(P_{c} \rightarrow \Lambda_{c}+\bar{D}\right)= & g_{\Lambda_{c} D N}^{2} g_{\psi^{\prime} D D}^{2} \frac{4 k E_{\Lambda_{c}} E_{D}}{M_{P_{c}}} \\
& \times \frac{1}{\left(2 M_{N}\right)\left(2 M_{\psi^{\prime}}\right)\left(2 E_{\Lambda_{c}}\right)\left(2 E_{D}\right)} \\
& \times \frac{M_{\Lambda_{c}}+E_{\Lambda_{c}}}{2 M_{N}} \bigvee_{f}|\mathcal{M}|_{i \rightarrow f}^{2} \\
\approx & 0.6 \mathrm{MeV}
\end{aligned}
$$

\section{Other open charm decays of hadrocharmonium pentaquark}

Calculations of other three decays of the hadrocharmonium pentaquark into the open charm states

$P_{c} \rightarrow \Sigma_{c}+\bar{D}, \quad P_{c} \rightarrow \Lambda_{c}+\bar{D}^{*}, \quad P_{c} \rightarrow \Sigma_{c}^{*}+\bar{D}$,

are similar to the calculations above. All these decays go via exchange by the lightest particle with an open charm, $D$-meson. Kinematics for all these decays was already considered above and we will not repeat this discussion.

The $P_{c} \rightarrow \Sigma_{c}+\bar{D}$ decay is described by the $D$-exchange diagram in Fig. 11, that is similar to the $D$-exchange for $P_{c} \rightarrow \Lambda_{c}+\bar{D}$. Effective mass of the exchanged $D$-meson in this decay is $M_{*}(D)=1005 \mathrm{MeV}$. The amplitude for this decay differs from the decay $P_{c} \rightarrow \Lambda_{c}+\bar{D}$ only by the

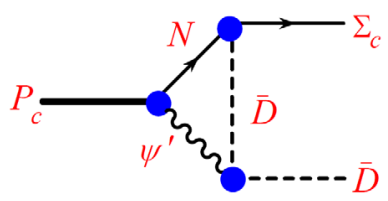

FIG. 11. Decay of hadrocharmonium pentaquark $P_{c}(4450)$ into states with open charm $\Sigma_{c}+\bar{D}$. 


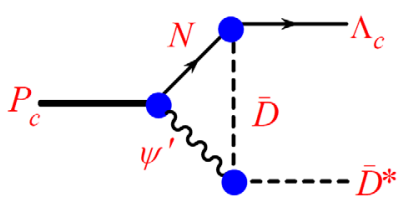

FIG. 12. Decay of hadrocharmonium pentaquark $P_{c}(4450)$ into states with open charm $\Sigma_{c}+\bar{D}^{*}$.

isospin factor that generates an enhancement factor 3 in the width. On the other hand the relationship between the coupling constants $g_{\Sigma_{c} N D}=g_{\Lambda_{c} N D} /(3 \sqrt{3})$ [see Eq. (A19) in Appendix A 2] supply a suppression factor for the $P_{c} \rightarrow$ $\Sigma_{c}+\bar{D}$ decay. After replacement of the coupling constants, masses and multiplication by 3 we can use Eq. (48) for calculation of the $P_{c} \rightarrow \Sigma_{c}+\bar{D}$ partial decay width. We obtain $\Gamma\left(P_{c} \rightarrow \Sigma_{c}+\bar{D}\right)=0.036 \mathrm{MeV}$, see Table IV. The suppression by an order of magnitude $\sim 1 / 9$ relative to the decay $P_{c} \rightarrow \Lambda_{c}+\bar{D}$ comes mainly from the ratio of the coupling constants squared times three from the isotopic factor, difference between the masses of $\Sigma_{c}$ and $\Lambda_{c}$ plays an insignificant role.

To calculate the partial decay width $P_{c} \rightarrow \Lambda_{c}+\bar{D}^{*}$ (see Fig. 12) we go through the by now standard steps: calculate the relativistic scattering amplitude $N+\psi^{\prime} \rightarrow \Lambda_{c}+\bar{D}^{*}$, make the nonrelativistic approximation for the constituent hadrons, derive an expression for the transition operator and calculate the decay amplitude. The sum of the matrix elements squared for the decay $P_{c} \rightarrow \Lambda_{c}+\bar{D}^{*}$ turns out to be

$$
\begin{aligned}
\mathcal{E}_{f}|\mathcal{M}|_{i \rightarrow f}^{2}= & \left|M_{c}\left(0, \frac{3}{2} \mid 0\right)\right|^{2}+5\left|M_{t}\left(0, \frac{3}{2} \mid 2\right)\right|^{2} \\
& +\frac{2\left(a_{1}+a_{2}\right)^{2} k^{2}}{3 M_{*}^{2}(D)}\left|M_{d}\left(0, \frac{3}{2} \mid 1\right)\right|^{2} \\
& +\frac{2 b(1+3 b) k^{4}}{M_{*}^{4}(D)}\left|M_{c}\left(0, \frac{3}{2} \mid 0\right)\right|^{2} \\
& +\frac{10 b k^{2}}{M_{*}^{2}(D)} M_{c}\left(0, \frac{3}{2} \mid 0\right) M_{t}\left(0, \frac{3}{2} \mid 0\right),
\end{aligned}
$$

where

$a_{1}=\frac{M_{N}-E_{\Lambda_{c}}}{M_{p s i^{\prime}}+M_{N}-M_{\Lambda_{c}}}, \quad a_{2}=1-\frac{2 M_{N}}{M_{N}+E_{\Lambda_{c}}}, \quad b=-a_{1} a_{2}$.

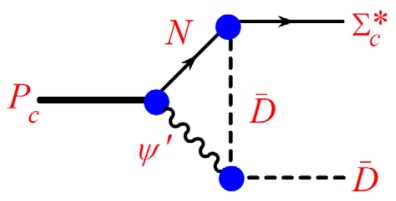

FIG. 13. Decay of hadrocharmonium pentaquark $P_{c}(4450)$ into states with open charm $\bar{D}+\Sigma_{c}^{*}$.

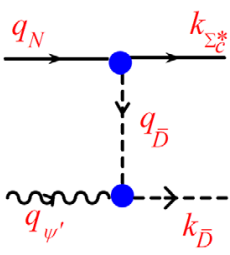

FIG. 14. Amplitude $N+\psi^{\prime} \rightarrow \Sigma_{c}^{*}+\bar{D}$.

The partial width is

$$
\begin{aligned}
\Gamma\left(P_{c} \rightarrow \Lambda_{c}+\bar{D}^{*}\right)= & g_{\Lambda_{c} N D}^{2} g_{\psi^{\prime} D D^{*}}^{2} \frac{4 k E_{\Lambda_{c}} E_{D^{*}}}{M_{P_{c}}} \\
& \times \frac{\left(M_{\psi^{\prime}}+M_{N}-E_{\Lambda_{c}}\right)^{2}}{\left(2 M_{N}\right)\left(2 M_{\psi^{\prime}}\right)\left(2 E_{\Lambda_{c}}\right)\left(2 E_{D^{*}}\right)} \frac{M_{\Lambda_{c}}+E_{\Lambda_{c}}}{2 M_{N}} \\
& \times \oiiint_{f}|\mathcal{M}|_{i \rightarrow f}^{2} \approx 4.2 \mathrm{MeV} .
\end{aligned}
$$

The $P_{c} \rightarrow \Sigma_{c}^{*}+\bar{D}$ decay goes via the $D$-exchange diagram in Fig. 13. The $\Sigma^{*} N D$ interaction Lagrangian (notice absence of $\gamma^{5}$ !) is in Table VI. We again go through the standard steps: calculate the relativistic scattering amplitude in Fig. 14, use this amplitude with the nonrelativistic initial particles to derive the transition operator, obtain the decay amplitude, sum matrix elements squared and calculate the decay width $\Gamma\left(P_{c} \rightarrow \Sigma_{c}^{*}+\bar{D}\right)=$ $0.42 \mathrm{MeV}$.

\section{DISCUSSION OF RESULTS}

We calculated the total and partial decay widths of the hadrocharmonium and molecular pentaquarks $P_{c}(4450)$ constructed in $[9,10]$. One could expect that decays into states with open charm dominate in the case of the molecular pentaquark, while the decay to $J / \psi N$ would be the dominant mode for the hadrocharmonium pentaquark, see discussion in the Introduction. The calculations above confirm these expectations both for the molecular

TABLE IV. Pentaquark $P_{c}(4450)$ decay widths in the hadrocharmonium picture.

\begin{tabular}{lcccc}
\hline \hline Decay mode & $L^{\mathrm{a}}$ & $k^{\mathrm{b}}(\mathrm{MeV})$ & $M_{*}(D)^{\mathrm{c}}(\mathrm{MeV})$ & $\Gamma^{\mathrm{d}}(\mathrm{MeV})$ \\
\hline$P_{c} \rightarrow J / \psi N$ & 0 & 820 & & 11 \\
$P_{c} \rightarrow \Lambda_{c} \bar{D}$ & 2 & 798 & 1133 & 0.6 \\
$P_{c} \rightarrow \Sigma_{c} \bar{D}$ & 2 & 529 & 1005 & 0.04 \\
$P_{c} \rightarrow \Lambda_{c} \bar{D}^{*}$ & 0,2 & 579 & 1218 & 4.2 \\
$P_{c} \rightarrow \Sigma_{c}^{*} \bar{D}$ & 0,2 & 360 & 959 & 0.4 \\
Total width & & & & 16.2 \\
\hline \hline
\end{tabular}

${ }^{\mathrm{a}}$ Lowest allowed orbital momentum.

${ }^{\mathrm{b}}$ Final momentum.

${ }^{c}$ Effective exchanged mass.

${ }^{\mathrm{d}}$ Decay width. 
and hadrocharmonium pentaquarks, see Tables I and IV. Total decay widths of the molecular and hadrocharmonium pentaquarks are comparable and are about a few dozen $\mathrm{MeV}$ in both scenarios. Taking into account uncertainties of the phenomenological coupling constants and unaccounted for relativistic corrections to the semirelativistic approximation used in the calculations these total widths are comfortably compatible with the width $\Gamma=39 \pm 5 \pm$ $19 \mathrm{MeV}$ measured experimentally [1,2].

We expect that the results for the relative magnitudes of partial decays widths in different open channels are more reliable than their absolute values. This happens because in the ratios of the partial widths values of the poorly known interaction constants often cancel and the ratios are more dependent on the matrix elements of the perturbation potentials between the initial and final wave functions. The partial decay width of the molecular pentaquark into the hidden charm states $J / \psi N$ is strongly suppressed, it is about one, two, or three orders of magnitude smaller than the partial widths for decays into different channels with open charm, see Table I. ${ }^{4}$ The suppression can be understood if we recall that the molecular pentaquark has a relatively large size, its root mean square radius is about $1.5 \mathrm{fm}$ [10]. To decay into states with hidden charm constituents of the molecular pentaquark need to exchange by a heavy charmed meson. In other words they should come very close to one another what is impeded by the large size of the loosely bound state wave function. The detailed considerations of the matrix elements in Sec. III C provide a quantitative justification for these conclusions.

The decay pattern of the hadrocharmonium pentaquark also looks as expected. The hadrocharmonium decays into states with open charm are suppressed in comparison with the hadrocharmonium decays into hidden charm states. Quantitatively this suppression is weaker than the suppression of the hidden charm decays in the case of the molecular pentaquark, compare the results in Tables I and IV. One of the partial widths for hadrocharmonium decay into open charm states $\left(P_{c} \rightarrow \Lambda_{c} \bar{D}\right)$ is only two and a half times smaller than the partial decay width to $J / \psi \bar{D}^{*}$. To decay into states with open charm constituents in the hadrocharmonium should come close to one another what happens when they exchange by a heavy charmed meson. The relatively weaker suppression of such hadrocharmonium processes in comparison with the respective molecular case decays is due to a larger binding energy and respectively smaller size (about $0.5 \mathrm{fm}$ ) of the hadrocharmonium bound state.

\footnotetext{
${ }^{4}$ Recent nonobservation of the pentaquark resonance in the formation reaction $\gamma+p \rightarrow J / \psi+p$ [27] could be interpreted as an indication of the molecular nature of the $\mathrm{LHCb}$ pentaquark. However, it is hard to reconcile this result with the initial $\mathrm{LHCb}$ discovery of the pentaquark in the invariant mass distribution of $J / \psi N$. Clearly more work is needed and it is too early to come to any definite conclusions.
}

We see that the decay patterns of the molecular and hadrocharmonium pentaquarks are vastly different. In the molecular scenario decays into $J / \psi$ are strongly suppressed, while the opposite happens in the hadrocharmonium case when a less pronounced suppression of decays into states with open charm is predicted. Total decay widths are comparable in both scenarios and are about a few dozen $\mathrm{MeV}$. Comparison of these decay patterns with the experimental data would hopefully help to reveal which of the two theoretical scenarios for pentaquarks (if either) is chosen by nature.

\section{ACKNOWLEDGMENTS}

This paper was supported by the NSF Grant No. PHY1724638.

\section{APPENDIX A: INTERACTION LAGRANGIANS AND INTERACTION CONSTANTS}

A number of phenomenological interaction Lagrangians was used in calculations in the main body of this paper. Coupling constants in these Lagrangians were discussed in the literature many times, see, e.g., [19,20,24,28-42] and references therein. There is no universal agreement on the values of some of these constants, while decay widths obtained above critically depend on these values. There are three groups of relevant Lagrangians that describe: (1) pion interaction with charmed hadrons, (2) $D$-boson interactions with baryons, and (3) $D$-boson interaction with heavy mesons. The interaction Lagrangians and coupling constants are collected in Tables V-VII. The interaction constants in these tables are known with vastly different degree of reliability. We tried to use the value of this or that constant obtained with a minimal number of theoretical assumptions. Below we discuss how these values arise and how accurate they are.

\section{Pion interaction constants in Table $\mathbf{V}$}

Pion interactions with heavy baryons and mesons are usually described in the framework of the heavy quark effective theory combined with the spontaneously broken $S U(3)_{L} \times S U(3)_{R}$ chiral symmetry of light quarks, see, e.g., [28-30] and references therein. It is worth mentioning that pion interactions can be formulated in the pseudoscalar and axial forms that are equivalent in the nonrelativistic limit. Connection between the respective coupling constants for the pion-nucleon interaction is provided by the classical Goldberger-Treiman relationship

$$
g_{\pi N N}=g_{N N}^{A} \frac{M_{N}}{F_{\pi}},
$$

where $g_{N N}^{A}$ is the nucleon axial charge and $g_{\pi N N}$ is the pseudoscalar interaction constant. 
TABLE V. Pion interactions.

\begin{tabular}{|c|c|c|}
\hline Interacting particles & Interaction Lagrangian & Coupling constant \\
\hline$\pi \Sigma_{c} \Lambda_{c}$ & $-i g_{\pi \Sigma_{c} \Lambda_{c}} \bar{\Lambda}_{c}^{\dagger} \gamma^{5} \Sigma_{c} \cdot \pi+$ H.c. & $g_{\pi \Sigma_{c} \Lambda_{c}}=19.2^{\mathrm{a}}$ \\
\hline$\pi \Sigma_{c} \Sigma_{c}$ & $-i g_{\pi \Sigma_{c} \Sigma_{c}} \epsilon_{a b c} \bar{\Psi}_{\Sigma}^{a} \gamma_{5} \Psi_{\Sigma}^{b} \pi^{c}+$ H.c. & $g_{\pi \Sigma_{c} \Sigma_{c}}=11.06^{\mathrm{b}}$ \\
\hline$\pi \Sigma_{c} \Sigma_{c}^{*}$ & $i \tilde{g}_{\pi \Sigma_{c} \Sigma_{c}^{*}} \bar{\Sigma}_{c}^{* \mu, a} \epsilon_{a b c} \Sigma_{c}^{b} \partial_{\mu} \pi^{c}+$ H.c. & 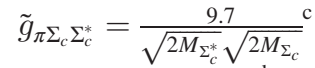 \\
\hline$\pi D D^{*}$ & $i g_{\pi D D^{*}}\left(D_{\mu}^{* \dagger} \partial^{\mu} \pi D-D^{\dagger} \partial^{\mu} \pi D_{\mu}^{*}\right)$ & $g_{\pi D D^{*}}=12.12^{\mathrm{d}}$ \\
\hline$\pi D^{*} D^{*}$ & $g_{\pi D^{*} D^{*}} \epsilon^{\mu \nu \alpha \beta} D_{\mu}^{* \dagger} \partial_{\nu} \pi \partial_{\alpha} D_{\beta}^{*}$ & $g_{\pi D^{*} D^{*}}=6.25 \mathrm{GeV}^{-1}$ \\
\hline \multicolumn{3}{|c|}{$\begin{array}{l}{ }^{\mathrm{a}} \text { From } \Gamma_{\exp }\left(\Sigma_{c}^{++} \rightarrow \Lambda_{c} \pi^{+}\right)=1.89_{-0.18}^{+0.09} \text { and } \Gamma\left(\Sigma_{c}^{0} \rightarrow \Lambda_{c} \pi^{-}\right)=1.83_{-0.19}^{+0.11} \mathrm{MeV} \text {, see Appendix A } 1 \text { and [19]. } \\
{ }^{\mathrm{b}} \text { See Appendix A } 1 . \\
{ }^{\mathrm{c}} \text { See Appendix A } 1 . \\
{ }^{\mathrm{d}} \text { From } \Gamma_{\exp }\left(D^{*+}(2010) \rightarrow D^{0} \pi^{+}\right)=56.5 \pm 0.1 \mathrm{keV} \text { and } \Gamma_{\exp }\left(D^{*+}(2010) \rightarrow D^{+} \pi^{0}\right)=25.6 \pm 0.6 \mathrm{keV} \text {, See } \\
\text { ppendix A } 1 \text { and [20]. }\end{array}$} \\
\hline
\end{tabular}

Relationships of this type exist not only for diagonal interactions but also for nondiagonal vertices, e.g., for the $\pi \Sigma_{c} \Lambda_{c}$ interaction. Axial form of the interaction is dictated by the Goldstone nature of pions and the axial charge can be calculated, at least in principle, see, e.g., [24,31].

Experimental data on the decay widths $\Sigma_{c}^{++} \rightarrow \Lambda_{c} \pi^{+}$ and $\Sigma_{c}^{0} \rightarrow \Lambda_{c} \pi^{-}$[43], provides direct access to the interaction constant $g_{\pi \Sigma_{c} \Lambda_{c}}$. With the Lagrangian in Table V one obtains

$$
\Gamma\left(\Sigma_{c} \rightarrow \Lambda_{c}+\pi\right)=\frac{g_{\pi \Sigma_{c} \Lambda_{c}}^{2}}{4 \pi} \frac{k\left(E_{\Lambda_{c}}-M_{\Lambda_{c}}\right)}{M_{\Sigma_{c}}},
$$

where $k$ is the decay momentum and $E_{\Lambda_{c}}$ is the energy of the final $\Lambda_{c}$. We obtain $g_{\pi \Sigma_{c} \Lambda_{c}}=19.3$ from the decay $\Sigma_{c}^{++} \rightarrow \Lambda_{c} \pi^{+}$and $g_{\pi \Sigma_{c} \Lambda_{c}}=19.1$ from the decay $\Sigma_{c}^{0} \rightarrow \Lambda_{c} \pi^{-}$. We used the average $g_{\pi \Sigma_{c} \Lambda_{c}}=19.2$ (compare $[19,28,32]$ ) in the calculations above.

There is no experimental data for the $\Sigma_{c} \Sigma_{c} \pi$ coupling, so we have chosen a roundabout way to determine the respective interaction constant. As mentioned above axial interaction constants can be in principle calculated theoretically if one knows form factors of the respective axial currents. Unfortunately, currently there is no effective way to calculate these form factors in QCD. ${ }^{5}$ It was suggested long time ago [31] to use the naive constituent quark model to calculate diagonal and transitional axial charges. The quark model predicts $g_{\Sigma_{c} \Lambda_{c}}^{a}=2 / \sqrt{3} \sim 1.154$ to be compared with the value we calculate from the experimental decay widths $g_{\Sigma_{c} \Lambda_{c}}^{a}=g_{\pi \Sigma_{c} \Lambda_{c}} F_{\pi} / M_{\Sigma_{c}} \sim 0.727$ (we neglect here mass difference of $\Sigma_{c}$ and $\Lambda_{c}$ ). It is clear that the accuracy of the quark model leaves much to be desired. We expect that it predicts ratios of axial constants more accurately than the axial constants themselves. The ratio of the axial constants $g_{\Sigma_{c} \Lambda_{c} \pi}^{a}$ and $g_{\Sigma_{c} \Sigma_{c}}^{a}$ in the quark model is $g_{\Sigma_{c} \Lambda_{c}}^{a} / g_{\Sigma_{c} \Sigma_{c}}^{a}=1 / \sqrt{3}$. The ratio of the respective

\footnotetext{
${ }^{5}$ It could be a good problem for the lattice gauge theory calculations.
}

pseudoscalar constants is proportional to the ratio of the axial constants and we obtain

$$
g_{\pi \Sigma_{c} \Sigma_{c}}=\frac{1}{\sqrt{3}} g_{\pi \Sigma_{c} \Lambda_{c}} \approx 11.0
$$

We used this value in calculations of the pentaquark decay widths. Other estimates of this constant $g_{\pi \Sigma_{c} \Sigma_{c}} \approx 10.76$ $[19,33]$ are based on the assumption that $g_{\pi \Sigma_{c} \Sigma_{c}}=g_{\pi \Sigma \Sigma}$. This value is consistent with our estimate.

The axial interaction Lagrangian $\Sigma_{c}^{*} \Sigma_{c} \pi$ is in Table V. There is no $\gamma^{5}$ in this Lagrangian since contraction of the positive-parity Rarita-Schwinger spin-vector $\bar{\Sigma}_{c}^{* \mu}$, spinor $\Sigma_{c}^{*}$ and the axial vector $\partial_{\mu} \pi$ is a true scalar. The interaction has the gradient form, and the dimensionful interaction constant is proportional to the respective transitional axial constant. Naive quark model $[28,32]$ predicts that ratio of the $\Sigma_{c}^{*} \Sigma_{c}$ and $\Sigma_{c} \Sigma_{c}$ axial charges is $\sqrt{3} / 2$. We parameterize the dimensionful interaction constant $\tilde{g}_{\pi \Sigma_{c} \Sigma_{c}^{*}}$ in terms of the dimensionless $g_{\pi \Sigma_{c} \Sigma_{c}^{*}}$

$$
\tilde{g}_{\pi \Sigma_{c} \Sigma_{c}^{*}}=\frac{g_{\pi \Sigma_{c} \Sigma_{c}^{*}}}{\sqrt{2 M_{\Sigma_{c}^{*}}} \sqrt{2 M_{\Sigma_{c}}}}
$$

and calculate its value

$$
g_{\pi \Sigma_{c} \Sigma_{c}^{*}}=\frac{\sqrt{3}}{2} \sqrt{\frac{M_{\Sigma_{c}^{*}}}{M_{\Sigma_{c}}}} g_{\pi \Sigma_{c} \Sigma_{c}} \approx 0.88 g_{\pi \Sigma_{c} \Sigma_{c}}=9.7 .
$$

This constant was used in calculations of the pentaquark decay width.

The constant $g_{\pi D D^{*}}$ is extracted from the experimental data on $\left(D^{*+}(2010) \rightarrow D^{0} \pi^{+}\right.$and $\left(D^{*+}(2010) \rightarrow D^{+} \pi^{0}\right)$ decays [43]. The decay width calculated with the Lagrangian in Table $\mathrm{V}$ is

$$
\Gamma\left(D^{*+}\right)_{\mathrm{tot}}=\frac{g_{\pi D D^{*}}^{2}}{8 \pi} \frac{k^{3}}{M_{D^{*}}^{2}}
$$


TABLE VI. Nucleon interactions.

\begin{tabular}{lcc}
\hline \hline Interacting particles & Interaction Lagrangian & Coupling constant \\
\hline$\Lambda_{c} N D$ & $i g_{\Lambda_{c} N D} \bar{N} \gamma^{5} \Lambda_{c} D+$ H.c. & $g_{\Lambda_{c} N D}=4.5$ \\
$\Sigma_{c} N D$ & $-i g_{\Sigma_{c} N D} \bar{N} \gamma^{5} \tau \cdot \Sigma_{c} D+$ H.c. & $g_{\Sigma_{c} N D}=0.9$ \\
$\Sigma_{c}^{*} N D$ & $g_{\Sigma_{c}^{*} N D} \bar{N}_{i} \tau_{i k}^{a} \Sigma_{c a}^{* \mu} \partial_{\mu} D_{k}^{\dagger}+$ H.c. & $g_{\Sigma_{c}^{*} N D}=0.55 \mathrm{GeV}^{-1}$ \\
\hline \hline
\end{tabular}

Combined with the experimental data this expression gives $g_{\pi D D^{*}}$ cited in Table V.

The constant $g_{\pi D^{*} D^{*}}$ can be obtained from $g_{\pi D D^{*}}$ using the heavy quark relationship (see, e.g., [34]) $g_{\pi D^{*} D^{*}}=$ $g_{\pi D D^{*}} / \sqrt{M_{D} M_{D^{*}}}$.

\section{Nucleon interactions}

\section{a. $\Lambda_{c} N D$ interaction and $\Lambda_{c}$ semileptonic decays}

Nucleon-charmed baryon- $D$-meson interaction constants were obtained in the literature from the $S U(4)$ invariant Lagrangians, see, e.g., [19,33,35], and references therein. The QCD sum rules were also used to obtain the value of $g_{\Lambda_{c} N D}$ [39-41], and produced $g_{\Lambda_{c} N D}=7.9 \pm 0.9$, what is significantly smaller than the $S U(4)$ prediction $g_{\Lambda_{c} N D}=$ -13.7 [35].

In view of such uncertainty we would like to go another route and connect the $D$-meson interaction constants with the experimental data on the weak semileptonic decay $\Lambda_{c} \rightarrow \Lambda+e^{+}+\nu_{e}$. The idea is to determine the constant $g_{\Lambda_{c} \Lambda D}$ from the experimental data on this decay and then use the $S U(3)$ flavor symmetry to calculate $g_{\Lambda_{c} N D}$ in terms of $g_{\Lambda_{c} \Lambda D}$.

Our approach to finding $g_{\Lambda_{c} \Lambda D}$ is similar to the Goldberger-Treiman derivation of the relationship between the pseudoscalar interaction constant $g_{\pi N N}$ and the nucleon axial charge in Eq. (A1). The decay $\Lambda_{c} \rightarrow \Lambda+e^{+}+\nu_{e}$ is described by six form factors

$$
\begin{aligned}
\left\langle\Lambda\left|\bar{s} \gamma^{\mu} c\right| \Lambda_{c}\right\rangle= & \bar{\Lambda}(p+q)\left[\gamma^{\mu} f_{1}\left(q^{2}\right)+i \sigma^{\mu \nu} q_{\nu} f_{2}\left(q^{2}\right)\right. \\
& \left.+q^{\mu} f_{3}\left(q^{2}\right)\right] \Lambda_{c}(p), \\
\left\langle\Lambda\left|\bar{s} \gamma^{\mu} \gamma^{5} c\right| \Lambda_{c}\right\rangle= & \bar{\Lambda}(p+q)\left[\gamma^{\mu} g_{1}\left(q^{2}\right)+i \sigma^{\mu \nu} q_{\nu} g_{2}\left(q^{2}\right)\right. \\
& \left.+q^{\mu} g_{3}\left(q^{2}\right)\right] \gamma^{5} \Lambda_{c}(p) .
\end{aligned}
$$

The transferred momentum squared $q^{2}$ is an invariant mass of the lepton pair and is kinematically bounded, $\sqrt{q^{2}} \leq M_{\Lambda_{c}}-M_{\Lambda}<M_{D}$. The lepton masses can be safely neglected in the theoretical description of the $\Lambda_{c} \rightarrow$ $\Lambda+e^{+}+\nu_{e}$ decay. Then the form factors $f_{3}$ and $g_{3}$ do not enter the decay amplitude due to conservation of the lepton currents.

The form factors have poles in $q^{2}$ at the masses of mesons with the respective quantum numbers but they are outside the kinematically allowed region. Let us calculate lowest mass pseudoscalar charmed meson $D$ contribution to the form factor $g_{3}$. We choose the pseudoscalar form for the $\Lambda_{c} \Lambda D$ interaction

$$
\mathcal{L}_{P}=i g_{\Lambda_{c} \Lambda D} \bar{\Lambda} \gamma^{5} \Lambda_{c} D
$$

and use the standard definition for the $D$-meson decay constant

$$
\left\langle 0\left|\bar{s} \gamma^{\mu} \gamma^{5} c\right| D(p)\right\rangle=-i f_{D} p^{\mu}
$$

where $f_{D} \approx 212 \mathrm{MeV}$ [43].

We approximate the pseudoscalar form factor of a pointlike axial current by the pole contribution

$$
g_{3}=\frac{f_{D} g_{\Lambda_{c} \Lambda D}}{M_{D}^{2}-q^{2}}
$$

and we would like to determine the constant $g_{\Lambda_{c} \Lambda D}$ from the experimental data on the semileptonic decay $\Lambda_{c} \rightarrow$ $\Lambda+e^{+}+\nu_{e}$. However, as mentioned above this form factor $g_{3}$ does not contribute to the $\Lambda_{c} \rightarrow \Lambda+e^{+}+\nu_{e}$ decay. To overcome this difficulty we consider the $c$-quark to be heavy enough to use the heavy quark approximation. According to the heavy quark theory only two of the six form factors describing a typical heavy-light transition in Eq. (A7) are independent (see, e.g., [44]), and

$$
f_{1}=g_{1}, \quad f_{2}=f_{3}=g_{2}=g_{3},
$$

Thus the form factors $g_{2}$ and $f_{2}$ coincide with the form factor $g_{3}$ in Eq. (A10). Numerous models for the form factors $f_{1}, f_{2}, g_{1}$, and $g_{2}$ were constructed in [45-50] and compared with the experimental data on the $\Lambda_{c} \rightarrow$ $\Lambda+e^{+}+\nu_{e}$ decay. Parametrizations of the form factors in these works depend on many parameters, and the simple pole ansatz in Eq. (A10) was never used. We considered the $q^{2}$-dependent form factors in [45-50] as experimental data and used the HQET relationships in Eq. (A11) to fit them not far from the pole with the simple pole ansatz in Eq. (A10). ${ }^{6}$ As a result of these fits we obtained approximate values of the coupling constant $g_{\Lambda_{c} \Lambda D}$.

\footnotetext{
${ }^{6}$ Some of the papers [45-50] where written before the branching ratio $\Gamma\left(\Lambda_{c} \rightarrow \Lambda e^{+} \nu\right) / \Gamma_{\text {tot }}$ changed from $2 \%$ to $3.6 \%$ [43]. To account for this change we rescaled the old results by the square root of the new and old branching ratios.
} 
The $S U(3)$ flavor symmetry of light quarks combined with the heavy quark theory provides a relationship between $g_{\Lambda_{c} \Lambda D}$ and $g_{\Lambda_{c} N D}$. Light quarks in $\Lambda_{c}$ are in the flavor antitriplet $\overline{\mathbf{3}}$ state, while $\Lambda$ is a member of the flavor octet $\mathbf{8}$, and the light quark in the current in Eq. (A7) (as well as in the $\bar{D}$-meson) is in the fundamental flavor representation 3. Then matrix elements of the flavor triplet $j_{\beta}$ currents between different flavor octet states and $\Lambda_{c}$ are proportional to the Clebsch-Gordon coefficients

$$
\left\langle H, a\left|j_{\beta}\right| H_{c}, \alpha\right\rangle \sim C_{\overline{\mathbf{3}} \alpha, 3 \beta}^{\mathbf{8} \alpha},
$$

where $a$ is an $S U(3)$ octet index, while $\alpha$ and $\beta$ are antitriplet and triplet indices, respectively. We use this relationship and Eq. (A7) to obtain

$$
g_{\Lambda_{c} N D}=\sqrt{\frac{3}{2}} g_{\Lambda_{c} \Lambda D}
$$

Fitting the form factors in [45-50] with the pole ansatz and using Eq. (A13) we obtained $g_{\Lambda_{c} N D}$ in the interval 3.5-5.5. These values are much smaller than $g_{\Lambda_{c} N D}=13.7$ [35] from the $S U(4)$ symmetry widely accepted in the literature. We think that [35] strongly overestimates $g_{\Lambda_{c} N D}$ and used $g_{\Lambda_{c} N D}=4.5$ in the calculations above. This is, of course, only a not too accurate estimate of this coupling constant.

\section{b. $\Sigma_{c} N D$ interaction and quark model}

We estimate the coupling constant $g_{\Sigma_{c} N D}$ using the constant $g_{\Lambda_{c} N D}$ from Eq. (A13). Unfortunately, there is no $S U$ (3) flavor relationship between $g_{\Sigma_{c} N D}$ and $g_{\Lambda_{c} N D}$ since light quarks in $\Sigma_{c}$ and $\Lambda_{c}$ are in different flavor representations ( $\mathbf{6}$ and $\overline{\mathbf{3}}$, respectively). One can obtain such a relationship in the constituent quark model. We start with the proton, $\Lambda_{c}, \Sigma_{c}$, and $D$ quark model wave functions. Quarks in a nucleon are in the antisymmetric color state and hence the remaining wave function is symmetric. It is a product of a symmetric coordinate wave function $f_{N}\left(\boldsymbol{r}_{1}, \boldsymbol{r}_{2}, \boldsymbol{r}_{3}\right)$ and a symmetric spin-flavor function. The proton wave function with spin up has the form (we suppress the antisymmetric color factor)

$$
\begin{aligned}
\Psi_{p}^{\uparrow}= & \frac{1}{3 \sqrt{2}}\left[2 u_{1}^{\uparrow} u_{2}^{\uparrow} d_{3}^{\downarrow}+2 u_{1}^{\uparrow} d_{2}^{\downarrow} u_{3}^{\uparrow}+2 d_{1}^{\downarrow} u_{2}^{\uparrow} u_{3}^{\uparrow}-u_{1}^{\downarrow} u_{2}^{\uparrow} d_{3}^{\uparrow}\right. \\
& -u_{1}^{\downarrow} d_{2}^{\uparrow} u_{3}^{\uparrow}-u_{1}^{\uparrow} u_{2}^{\downarrow} d_{3}^{\uparrow}-d_{1}^{\uparrow} u_{2}^{\downarrow} u_{3}^{\uparrow}-u_{1}^{\uparrow} d_{2}^{\uparrow} u_{3}^{\downarrow} \\
& \left.-d_{1}^{\uparrow} u_{2}^{\uparrow} u_{3}^{\downarrow}\right] f_{N}\left(\boldsymbol{r}_{1}, \boldsymbol{r}_{2}, \boldsymbol{r}_{3}\right) .
\end{aligned}
$$

Respectively, the $\Lambda_{c}$ and $\Sigma_{c}^{++}$wave functions (again with spin up) are

$$
\begin{gathered}
\Psi_{\Lambda_{c}}^{\uparrow}=\frac{1}{2} c_{1}^{\uparrow}\left[u_{2}^{\uparrow} d_{3}^{\downarrow}+d_{2}^{\downarrow} u_{3}^{\uparrow}-u_{2}^{\downarrow} d_{3}^{\uparrow}-d_{2}^{\uparrow} u_{3}^{\downarrow}\right] f_{\Lambda_{c}}\left(\boldsymbol{r}_{1}, \boldsymbol{r}_{2}, \boldsymbol{r}_{3}\right), \\
\Psi_{\Sigma_{c}^{++}}^{\uparrow}=\frac{1}{\sqrt{6}}\left[2 c_{1}^{\downarrow} u_{2}^{\uparrow} u_{3}^{\uparrow}-c_{1}^{\uparrow} u_{2}^{\downarrow} u_{3}^{\uparrow}-c_{1}^{\uparrow} u_{2}^{\uparrow} u_{3}^{\downarrow}\right] f_{\Sigma_{c}}\left(\boldsymbol{r}_{1}, \boldsymbol{r}_{2}, \boldsymbol{r}_{3}\right),
\end{gathered}
$$

where the coordinate wave functions $f_{\Lambda_{c}}\left(\boldsymbol{r}_{1}, \boldsymbol{r}_{2}, \boldsymbol{r}_{3}\right)$ and $f_{\Sigma_{c}}\left(\boldsymbol{r}_{1}, \boldsymbol{r}_{2}, \boldsymbol{r}_{3}\right)$ are symmetric with respect to the permutation $\boldsymbol{r}_{2} \leftrightarrow \boldsymbol{r}_{3}$. The $D^{0}$-meson wave function is

$$
\Psi_{D}^{0}=\frac{1}{\sqrt{2}}\left[c_{1}^{\downarrow} \bar{u}_{2}^{\uparrow}+c_{1}^{\uparrow} \bar{u}_{2}^{\downarrow}\right] f_{D}\left(\boldsymbol{r}_{1}, \boldsymbol{r}_{2}\right) .
$$

Transitions $\Lambda_{c} \rightarrow N+D$ and $\Sigma_{c} \rightarrow N+D$ in the quark model happen when a heavy $c$-quark emits a hard gluon that creates a light quark-antiquark pair. The heavy spectator $c$-quark picks up the light antiquark and forms $D$-meson, and the light quark joins the remaining two light quarks to form a nucleon. Emission of a hard gluon followed by the creation of a light quark-antiquark pair is effectively described by a flavor singlet operator $S$. Hence, the coupling constants $g_{\Sigma_{c} N D}$ and $g_{\Lambda_{c} N D}$ are proportional to the overlap integrals

$$
g_{\Sigma_{c} N D}=\left\langle D N|S| \Lambda_{c}\right\rangle, \quad g_{\Lambda_{c} N D}=\left\langle D N|S| \Sigma_{c}\right\rangle .
$$

We assume that the coordinate wave functions $f_{\Lambda_{c}}\left(\boldsymbol{r}_{1}, \boldsymbol{r}_{2}, \boldsymbol{r}_{3}\right)$ and $f_{\Sigma_{c}}\left(\boldsymbol{r}_{1}, \boldsymbol{r}_{2}, \boldsymbol{r}_{3}\right)$ coincide. Then

$$
g_{\Sigma_{c} N D}=\frac{1}{6} g, \quad g_{\Lambda N D}=\sqrt{\frac{3}{2}} g,
$$

where $g$ is one and the same overlap integral of the coordinate wave functions.

Thus we obtain the quark model prediction

$$
g_{\Sigma_{c} N D}=\frac{g_{\Lambda_{c} N D}}{3 \sqrt{3}} .
$$

Numerically, $g_{\Sigma_{c} N D} \approx 1.35$ what is again less than $g_{\Sigma_{c} N D}=$ 2.69 used in the literature, see, e.g., [33].

\section{c. $\Sigma_{c}^{*} N D$ interaction and heavy quark theory}

We consider $c$-quark as a heavy quark and use the heavy quark theory to connect coupling constants of the $\Sigma_{c} N D$ and $\Sigma_{c}^{*} N D$ interactions. Due to the heavy quark spin symmetry heavy-light isodoublet mesons $(c \bar{q})$, namely the pseudoscalar $D$-meson with spin zero and the vector $D^{*}$-meson with spin one form a spin doublet. This doublet in the covariant notation can be written as a two-index matrix field

$$
H^{(v)}(x)=\frac{1+\not p}{2}\left[\not^{*(v)}+i D^{(v)} \gamma^{5}\right],
$$


where $v^{\mu}$ is the heavy quark four-velocity, and $D^{(v)}$ and $D_{\mu}^{*(v)}\left(v^{\mu} D_{\mu}^{*(v)}=0\right)$ are pseudoscalar and transverse vector field, respectively. The first index of the two-index matrix field $H^{(v)}$ is the spinor index of the heavy $c$-quark and the second is spinor index of the light quark (for notation and more details see [44]). The field $H^{(v)}(x)$ transforms bilinearly under the Lorentz transformations.

Spin of light quarks in the isotriplet heavy baryons $(c q q)$ is one and these baryons form a spin doublet with spins $1 / 2$ and $3 / 2$. This doublet is described by the heavy quark theory field

$$
S_{\mu}^{(v)}=-\frac{1}{\sqrt{3}}\left(\gamma_{\mu}+v_{\mu}\right) \gamma^{5} \Sigma_{c}^{(v)}+\Sigma_{c \mu}^{*(v)},
$$

where the $\Sigma_{c}^{(v)}$ and $\Sigma_{c \mu}^{*(v)}$ are spinor and Rarita-Schwinger fields, respectively. Both fields satisfy the heavy quark theory Dirac equations $\not \Sigma_{c}^{(v)}=\Sigma_{c}^{(v)}$ and $\not \Sigma_{c \mu}^{(v)}=\Sigma_{c \mu}^{(v)}$. The Rarita-Schwinger field satisfies also the standard additional conditions $v^{\mu} \Sigma_{c \mu}^{*(v)}=\gamma^{\mu} \Sigma_{c \mu}^{*(v)}=0$, that are necessary to reduce the number of independent components of the field describing the particle with spin $3 / 2$ to four. Easy to see that due to transversality of the field $\Sigma_{c \mu}^{*(v)}$ the spin-doublet field $S_{\mu}^{(v)}$ satisfies the condition $v^{\mu} S_{\mu}^{(v)}=0$.

The simplest interaction Lagrangian preserving all symmetries of the strong interactions has the form

$$
\mathcal{L}_{P}=i g \bar{S}_{\nu}^{(v)} \sigma^{\mu \nu} \gamma^{5} H^{(v)} \partial_{\nu} N+\text { H.c. },
$$

where $N$ is the four-component nucleon field.

In the logic of the heavy quark theory interaction with light degrees of freedom should not change velocity of the heavy quark, and emission of a light nucleon with small but nonzero velocity should be considered as a first order correction to the heavy quark limit. This explains why the derivative in the interaction Lagrangian in Eq. (A22) is applied to the nucleon field, what makes the interaction vertex proportional to the nucleon velocity. The interaction Lagrangian in Eq. (A22) is therefore by construction a first order correction to the heavy quark limit and we avoid a hard task of calculating corrections on the background of large zero order term contributions.

We are looking for a relationship between the $\Sigma_{c} N D$ and $\Sigma_{c}^{*} N D$ interaction constants so the term with $D^{*}$ in Eq. (A22) can be omitted, and effectively

$$
H^{(v)} \rightarrow \frac{1+\not p}{2} i D^{(v)} \gamma^{5}
$$

Then after substitution of the explicit expression for the field $\bar{S}_{\mu}^{(v)}$ in Eq. (A22) one obtains

$$
\begin{aligned}
\mathcal{L}_{P} \rightarrow & i g\left[\frac{1}{\sqrt{3}} \bar{\Sigma}_{c}^{(v)} \gamma^{5}\left(\gamma_{\mu}+v_{\mu}\right)+\bar{\Sigma}_{c \mu}^{*(v)}\right] \sigma^{\mu \nu} \frac{1-\not p}{2} i D^{(v)} \partial_{\nu} N \\
& + \text { H.c. }
\end{aligned}
$$

The heavy quark theory $\Sigma_{c}^{*} N D$ interaction term turns into

$$
\mathcal{L}_{\Sigma_{c}^{*} N D}=-i g \bar{\Sigma}_{c \mu}^{*(v)} \partial^{\mu} D N+\text { H.c. },
$$

In the transformations leading to this expression we used the conditions on the field $\Sigma_{c \mu}^{*(v)}$ below Eq. (A21), the explicit expression $\sigma^{\mu \nu}=i\left(\gamma^{\mu} \gamma^{\nu}-g^{\mu \nu}\right)$, and allowed ourselves integration by parts. Obviously this heavy quark theory interaction coincides with the respective effective Lagrangian in Table VI, and, hence $g_{\Sigma_{c}^{*} N D}=g$.

Similar calculations with the field $\bar{\Sigma}_{c}^{(v)}$ lead to the heavy quark theory $\Sigma_{c} N D$ interaction term

$$
\mathcal{L}_{\Sigma_{c} N D}=i g \sqrt{3} \bar{\Sigma}_{c}^{(v)} \gamma^{5} v^{\nu} D^{(v)} \partial_{\nu} N+\text { H.c. }
$$

As discussed above this Lagrangian is a first order correction to the heavy quark limit due to the explicit derivative of the light nucleon field. Hence, it is legitimate to let $v^{\mu}=(1, \mathbf{0})$ in all other terms. Then only the time derivative proportional to the light nucleon mass survives in the expression above, and the interaction term in Eq. (A26) coincides with the respective phenomenological Lagrangian in Table VI, and we conclude that (recall that $g_{\Sigma_{c}^{*} N D}=g$ )

$$
g_{\Sigma_{c}^{*} N D}=\frac{g_{\Sigma_{c} N D}}{\sqrt{3} M_{N}} .
$$

We use $g_{\Sigma_{c} N D}$ calculated above and obtain $g_{\Sigma_{c}^{*} N D}=$ $0.55 \mathrm{GeV}^{-1}$. This value is much smaller than $g_{\Sigma_{c}^{*} N D}=$ $6.5 \mathrm{GeV}^{-1}$ cited in [19]. The authors of [19] made an assumption that $g_{\Sigma_{c}^{*} N D}=g_{\Sigma^{*} N K}$. Thus assumption can be justified in the framework of the heavy quark symmetry if one considers both the $s$ - and $c$-quarks as heavy quarks. In its turn $g_{\Sigma^{*} N K}$ was calculated in $[37,38]$ from $S U(3)$ flavor symmetry. The value of $g_{\Sigma_{c}^{*} N D}$ obtained above is only an estimate but we expect it to be more reliable than the one in [19] since simultaneous use of the $S U(3)$ flavor symmetry and heavy quark theory for $s$ - and $c$-quarks hardly can be justified.

\section{Charmonium interactions}

Generalized vector dominance and/or QCD sum rules can be used to calculate $J / \psi$ and $\psi^{\prime}$ interaction constants with $D$ meson, see, e.g., [42] for a review. The basic assumption of the generalized vector dominance is that photon interacts with $D$ via transitions into virtual vector mesons. Consider vector meson $V$ that is a bound state of $c \bar{c}$ quarks. The zero component of the $c$-quark electric 
TABLE VII. Charmonium interactions.

\begin{tabular}{lcc}
\hline \hline Interacting particles & Interaction Lagrangian & Coupling constant \\
\hline$J / \psi D D$ & $-i g_{\Psi D D} \psi_{\mu}\left(\partial_{\mu} D D^{\dagger}-D \partial_{\mu} D^{\dagger}\right)$ & $g_{D D J / \psi}=7.44^{\mathrm{a}}$ \\
$\psi^{\prime} D D$ & $-i g_{\Psi^{\prime} D D} \psi_{\mu}^{\prime}\left(\partial_{\mu} D D^{\dagger}-D \partial_{\mu} D^{\dagger}\right)$ & $g_{D D \psi^{\prime}}=12.51^{\mathrm{b}}$ \\
$J / \psi D D^{*}$ & $-g_{J / \psi D^{*} D} \epsilon^{\mu \nu \alpha \beta} \partial_{\mu} \psi_{\nu}\left(D_{\alpha}^{* \dagger} \stackrel{\leftrightarrow}{\partial}_{\beta} D-D^{\dagger} \stackrel{\leftrightarrow}{\partial}_{\beta} \bar{D}_{\alpha}^{*}\right)$ & $g_{J / \psi D^{*} D}=2.49 \mathrm{GeV}^{-1 \mathrm{c}}$ \\
$\psi^{\prime} D D^{*}$ & $-g_{\psi^{\prime} D^{*} D^{\mu}} \epsilon^{\mu \nu \alpha \beta} \partial_{\mu} \psi_{\nu}^{\prime}\left(D_{\alpha}^{* \dagger} \stackrel{\leftrightarrow}{\partial}_{\beta} D-D^{\dagger} \stackrel{\leftrightarrow}{\partial}_{\beta} \bar{D}_{\alpha}^{*}\right)$ & $g_{\psi^{\prime} D D^{*}}=3.52 \mathrm{GeV}^{-1 \mathrm{~d}}$ \\
\hline \hline
\end{tabular}

${ }^{\mathrm{a}}$ Generalized vector dominance, see Appendix A 3 and [36].

${ }^{\mathrm{b}}$ Generalized vector dominance, see Appendix A 3 and [36].

${ }^{\mathrm{c}}$ Generalized vector dominance and heavy quarks symmetry, see Appendix A 3 and [36].

${ }^{\mathrm{d}}$ Generalized vector dominance and heavy quarks symmetry, see Appendix A 3 and [36].

current $j_{(c)}^{\mu}=Q_{c} \bar{c} \gamma^{\mu} c\left(Q_{c}\right.$ is the $c$-quark charge) measures electric charge of the $c$-quark in $D$ meson. At zero momentum transfer $\left\langle D\left|j_{(c)}^{0}\right| D\right\rangle \sim Q_{c}$. On the other hand due to vector dominance the same matrix element is proportional to $g_{D V D}\left(1 / M_{V}^{2}\right) Q_{c} f_{V} M_{V}$, where $M_{V}$ is the vector meson mass and its decay constant $f_{V}$ is defined by the relationship $\left\langle 0\left|\bar{c} \gamma^{\mu} c\right| V\right\rangle=f_{V} M_{V} \epsilon^{\mu}$. Comparing these two expressions for the current matrix element we obtain $g_{D D V}=M_{V} / f_{V}$. The vector meson decay constant $f_{V}$ is determined from the partial decay width

$$
\Gamma\left(V \rightarrow e^{+} e^{-}\right)=\frac{4 \pi \alpha^{2}}{3} \frac{f_{V}^{2} Q_{c}^{2}}{M_{V}},
$$

and

$$
f_{V}=\frac{1}{2 \alpha Q_{c}} \sqrt{\frac{3 M_{V} \Gamma\left(V \rightarrow e^{+} e^{-}\right)}{\pi}} .
$$

Experimentally $\Gamma\left(\mathrm{J} / \psi \rightarrow e^{+} e^{-}\right)=5.55 \pm 0.14 \pm 0.02 \mathrm{keV}$ and $\Gamma\left(\psi^{\prime} \rightarrow e^{+} e^{-}\right)=2.33 \pm 0.04 \mathrm{keV}$ [43]. Then $f_{\psi} \approx$ $416.3 \mathrm{MeV}$ and $f_{\psi^{\prime}} \approx 294.68 \mathrm{MeV}$ [34], and

$$
g_{J / \psi D D}=\frac{M_{\psi}}{f_{\psi}}=7.44, \quad g_{\psi^{\prime} D D}=\frac{M_{\psi^{\prime}}}{f_{\psi^{\prime}}}=12.51 .
$$

The dimensionful constants $g_{\psi^{\prime} D D^{*}}$ and $g_{J / \psi D D^{*}}$ are calculated from the heavy quark relationships (see, e.g., [36])

$$
\begin{gathered}
g_{J / \psi D D^{*}}=\frac{g_{J / \psi D D}}{M_{J / \psi}} \sqrt{\frac{M_{D^{*}}}{M_{D}}}, \\
g_{\psi^{\prime} D D^{*}}=\frac{g_{\psi^{\prime} D D}}{M_{\psi^{\prime}}} \sqrt{\frac{M_{D^{*}}}{M_{D}}} .
\end{gathered}
$$

[1] R. Aaij et al. (LHCb Collaboration), Phys. Rev. Lett. 115, 072001 (2015).

[2] R. Aaij et al. (LHCb Collaboration), Phys. Rev. Lett. 117, 082003 (2016); 117, 109902(E) (2016); 118, 119901 (2017).

[3] R. F. Lebed, R. E. Mitchell, and E. S. Swanson, Prog. Part. Nucl. Phys. 93, 143 (2017).

[4] A. Ali, J. S. Lange, and S. Stone, Prog. Part. Nucl. Phys. 97, 123 (2017).

[5] A. Esposito, A. Pilloni, and A. D. Polosa, Phys. Rep. 668, 1 (2017).

[6] S. L. Olsen, T. Skwarnicki, and D. Zieminska, Rev. Mod. Phys. 90, 015003 (2018).

[7] F.-K. Guo, C. Hanhart, U.-G. Meißsner, Q. Wang, Q. Zhao, and B.-S. Zou, Rev. Mod. Phys. 90, 015004 (2018).

[8] M. Karliner, J. L. Rosner, and T. Skwarnicki, Annu. Rev. Nucl. Part. Sci. 68, 17 (2018).
[9] M. I. Eides, V. Yu. Petrov, and M. V. Polyakov, Phys. Rev. D 93, 054039 (2016).

[10] M. I. Eides, V. Yu. Petrov, and M. V. Polyakov, Eur. Phys. J. C 78, 36 (2018).

[11] M. N. Anwar, M. A. Bedolla, J. Ferretti, and E. Santopinto, arXiv:1807.01207.

[12] A. Sibirtsev and M. B. Voloshin, Phys. Rev. D 71, 076005 (2005).

[13] S. Dubynskiy and M. B. Voloshin, Phys. Lett. B 666, 344 (2008).

[14] X. Li and M. B. Voloshin, Mod. Phys. Lett. A 29, 1450060 (2014).

[15] S. J. Brodsky, I. Schmidt, and G. F. de Teramond, Phys. Rev. Lett. 64, 1011 (1990).

[16] M. Luke, A. V. Manohar, and M. J. Savage, Phys. Lett. B 288, 355 (1992).

[17] M. B. Voloshin, Prog. Part. Nucl. Phys. 61, 455 (2008). 
[18] M. B. Voloshin and L. B. Okun, Pis'ma Zh. Eksp. Teor. Fiz. 23, 369 (1976) [JETP Lett. 23, 333 (1976)].

[19] Y.-H. Lin, C.-W. Shen, F.-K Guo, and B.-S. Zou, Phys. Rev. D 95, 114017 (2017).

[20] C.-W. Shen, F.-K. Guo, J.-J. Xie, and B.-S. Zou, Nucl. Phys. A954, 393 (2016).

[21] Q.-F Lü and Y.-B. Dong, Phys. Rev. D 93, 074020 (2016).

[22] C. W. Shen and Y. H. Lin, Phys. Part. Nucl. Lett. 15, 402 (2018).

[23] K. Azizi, Y. Sarac, and H. Sundu, Phys. Lett. B 782, 694 (2018).

[24] J. Haidenbauer, S. Petschauer, N. Kaiser, U.-G. Meißner, and W. Weise, Eur. Phys. J. C 77, 760 (2017).

[25] N. A. Törnqvist, Phys. Rev. Lett. 67, 556 (1991).

[26] L. D. Landau and E. M. Lifshitz, Quantum Mechanics, 3rd ed. (Butterworth-Heinemann, Amsterdam, 2003).

[27] E. Chudakov on behalf of the GlueX Collaboration, Proceedings of the 23d International Spin Symposium, Ferrara (2018), http://spin2018.unife.it/.

[28] T.-M. Yan, H.-Y. Cheng, Ch.-Y. Cheung, G.-L. Lin, Y. C. Lin, and H.-L. Yu, Phys. Rev. D 46, 1148 (1992); 55, 5851(E) (1997).

[29] P. Cho, Phys. Lett. B 285, 145 (1992).

[30] Y. Shimizu, D. Suenaga, and M. Harada, Phys. Rev. D 93, 114003 (2016).

[31] D. O. Riska and G. E. Brown, Nucl. Phys. A679, 577 (2001).

[32] Y.-R. Liu and M. Oka, Phys. Rev. D 85, 014015 (2012).

[33] E. J. Garzon and J.-J. Xie, Phys. Rev. C 92, 035201 (2015).

[34] D.-Y. Chen and Y.-B. Dong, Phys. Rev. D 93, 014003 (2016).

[35] W. Liu, C. M. Ko, and Z. W. Lin, Phys. Rev. C 65, 015203 (2001).
[36] D.-Y. Chen, X. Liu, and T. Matsuki, Phys. Rev. D 88, 014034 (2013).

[37] Y. Oh, C. M. Ko, and K. Nakayama, Phys. Rev. C 77, 045204 (2008).

[38] M. Döring, C. Hanhart, F. Huangb, S. Krewalda, U.-G. Meißner, and D. Rönchen, Nucl. Phys. A851, 58 (2011).

[39] F. S. Navarra and M. Nielsen, Phys. Lett. B 443, 285 (1998).

[40] F. S. Navarra and M. Nielsen, Nucl. Phys. B, Proc. Suppl. 74, 214 (1999).

[41] F. O. Durães, F. S. Navarra, and M. Nielsen, Phys. Lett. B 498, 169 (2001).

[42] M. E. Bracco, M. Chiapparini, F. S. Navarra, and M. Nielsen, Prog. Part. Nucl. Phys. 67, 1019 (2012).

[43] M. Tanabashi et al. (Particle Data Group), Phys. Rev. D 98, 030001 (2018).

[44] A. V. Manohar and M. B. Wise, Heavy Quark Physics (Cambridge University Press, Cambridge, England, 2000).

[45] J. W. Hinson et al. (CLEO Collaboration), Phys. Rev. Lett. 94, 191801 (2005).

[46] Y.-L. Liu, M.-Q. Huang, and D.-W. Wang, Phys. Rev. D 80, 074011 (2009).

[47] T. Gutsche, M. A. Ivanov, J. G. Körner, V. E. Lyubovitskij, and P. Santorelli, Phys. Rev. D 93, 034008 (2016).

[48] R. N. Faustov and V. O Galkin, Eur. Phys. J. C 76, 628 (2016).

[49] C.-F. Li, Y.-L. Liu, K. Liu, C.-Y. Cui, and M.-Q. Huang, J. Phys. G 44, 075006 (2017).

[50] M. M. Hussain and W. Roberts, Phys. Rev. D 95, 053005 (2017). 